\title{
Le Chevalier au Cygne à la fin du Moyen Âge
}

Renouvellements, en vers et en prose, de l'épopée romanesque des origines de Godefroy de Bouillon

\section{Catherine Gaullier-Bougassas}

\section{(2) OpenEdition}

12 Journals

Édition électronique

URL : https://journals.openedition.org/crm/2232

DOI : $10.4000 / \mathrm{crm} .2232$

ISSN : 1955-2424

Éditeur

Honoré Champion

\section{Édition imprimée}

Date de publication : 30 décembre 2005

Pagination : 115-146

ISSN : $1272-9752$

\section{Référence électronique}

Catherine Gaullier-Bougassas, "Le Chevalier au Cygne à la fin du Moyen Âge », Cahiers de recherches

médiévales [En ligne], 12 | 2005, mis en ligne le 30 décembre 2008, consulté le 15 décembre 2022.

URL : http://journals.openedition.org/crm/2232 ; DOI : https://doi.org/10.4000/crm.2232

Ce document a été généré automatiquement le 15 décembre 2022.

Tous droits réservés 


\section{Le Chevalier au Cygne à la fin du Moyen Âge}

Renouvellements, en vers et en prose, de l'épopée romanesque des origines de Godefroy de Bouillon

\section{Catherine Gaullier-Bougassas}

1 Les premières chansons de croisade, la Chanson d'Antioche, la Conquête de Jérusalem et les Chétifs, suscitent tout au long du XIII ${ }^{e}$ siècle l'écriture de plusieurs autres poèmes, jusqu'à la constitution d'un cycle qui prend une dimension généalogique nouvelle. L'épopée de la première Croisade se trouve alors inscrite au cœur d'un récit sur les origines et le devenir d'un lignage et d'un seul, celui de Godefroy de Bouillon, fondateur de la première dynastie des rois de Jérusalem. Des récits, ajoutés les uns après les autres avant la Chanson d'Antioche, forment l'ouverture du cycle: la Naissance du Chevalier au Cygne (versions Elioxe et Beatrix), le Chevalier au Cygne, la Fin d'Elias, les Enfances Godefroi et le Retour de Cornumaran. Ils se consacrent aux origines mythiques de la famille et au début de la carrière de Godefroy ${ }^{1}$, tandis qu'à l'autre extrémité du cycle des continuations prolongent la Chanson de Jérusalem jusqu'à la mort de Godefroy, puis retracent le règne de son frère et de ses descendants. Elles s'arrêtent à la mort de Baudouin IV, le roi lépreux, et aux victoires qui lui sont prêtées contre Saladin, comme si les auteurs n'avaient pas voulu retracer la perte de Jérusalem par les Croisés². L'axe généalogique contribue alors à donner une unité au cycle, qui se donne à lire autant comme un «monument » à la gloire du lignage de Bouillon que comme une épopée des croisades : on pourrait ainsi comprendre pourquoi la seconde Croisade, dépourvue d'incidence sur l'histoire du royaume de Jérusalem, a été omise. Quant à la fin du cycle, elle coïncide avec une rupture dans la succession des rois de Jérusalem : la famille de Bouillon perd le contrôle du royaume de Jérusalem puis des dernières possessions chrétiennes en Orient, remplacée par d'autres lignages qui ne parviendront jamais à s'assurer une maîtrise de la Terre sainte. Les Lusignan en constituent peut-être le plus important, même si, après la défaite de Gui de Lusignan contre Saladin, ils ne règnent plus sur la ville. Au XIII ${ }^{e}$ siècle, ils se réapproprient néanmoins le titre de roi de Jérusalem, qu'ils conservent jusqu'à la fin du Moyen Âge, et on sait qu'au XIV ${ }^{e}$ siècle ils inspirent à leur tour l'écriture d'une chronique sous forme d'épopée, la Prise 
d'Alexandrie de Guillaume de Machaut, qui magnifie la « geste » de Pierre I $^{\text {er }}$ de Chypre, puis celle des Romans de Mélusine de Jean d'Arras et de Coudrette. À la différence des poèmes du premier cycle de la croisade, ces œuvres n'ont jamais été réunies. La chronique épique des croisades de Pierre Ir $^{\text {rr }}$ 'évoque d'ailleurs pas la légende des origines féeriques du lignage, qui devait pourtant déjà circuler, au moins sous des formes orales, et les auteurs des "romans historiques", en dépit de brèves allusions à la mort de ce roi de Chypre Lusignan, ignorent l'ouvrage de l'autorité illustre qu'était pourtant Guillaume de Machaut au XIV ${ }^{\mathrm{e}}$ siècle $^{3}$.

2 La volonté d'une glorification du lignage de Bouillon organise de toute évidence les poèmes relatifs au Chevalier au Cygne et il est probable qu'à la fin du Moyen Âge, les auteurs des Romans de Mélusine se sont souvenus de ce modèle d'ancêtre féerique pour célébrer la famille de Lusignan et leurs descendants présumés. Si les poèmes de la première Croisade magnifient une Histoire authentique avec les procédés d'agrandissement et d'embellissement propres à l'épopée, si la Chanson d'Antioche et la Conquête de Jérusalem peuvent se définir comme des "épopées historiques », le travail de la fiction sur lequel repose la légende du Chevalier au Cygne n'infirme en aucun cas l'authenticité que les auteurs revendiquent. À la différence des romans généalogiques anglo-normands de la même époque, la Naissance du Chevalier au Cygne et le Chevalier au Cygne célèbrent d'ailleurs l'ancêtre supposé d'un personnage historique bien connu et, pour ce faire, ils ne remontent pas dans un passé très lointain : grand-père de Godefroy, le Chevalier au Cygne est supposé appartenir à un temps relativement proche.

3 L'existence de la légende de l'ascendance féerique des Bouillon est attestée dès la fin du XII ${ }^{e}$ siècle, puisqu'une lettre de Guy de Bazoches l'évoque vers 1175, tandis que, quelques années plus tard, Guillaume de Tyr la rejette comme fabuleuse ${ }^{4}$. Toujours à la fin du XII ${ }^{e}$ siècle, la Chanson d'Antioche rappelle comme des faits bien connus le séjour $\mathrm{du}$ Chevalier au Cygne à Nimègue et son mariage avec la duchesse de Bouillon, sans évoquer sa naissance (v. 1451-1472). C'est ensuite le conte folklorique des enfantscygnes qui a été annexé à l'histoire du lignage : si Jean de Haute-Seille le relate à la fin du XII ${ }^{e}$ siècle sans le relier à la famille de Bouillon, son adaptateur en romanz, Herbert, évoque comme une vérité établie une filiation désormais bien attestée au début du $\mathrm{XIII}^{\mathrm{e}}$ siècle ${ }^{5}$. G. Duby a analysé comment l'écriture de récits généalogiques, en plein essor au XII siècle, aussi bien en latin qu'en français, a pu susciter l'invention d'ancêtres féeriques, dans des chroniques familiales écrites en latin et commandées par des mécènes aristocratiques ${ }^{6}$. Divers récits latins du XII ${ }^{e}$ et XIII ${ }^{e}$ siècles retracent aussi la tentative d'intégration d'une fée dans le monde des hommes et son échec, annonçant l'histoire de la fée Mélusine ${ }^{7}$. Marie de France donne enfin à l'un de ses lais les plus célèbres, Yonec, une valeur généalogique en imaginant un chevalier-oiseau comme ancêtre des seigneurs de Bretagne. Dans le même temps, de la fin du XII siècle au milieu du XIII ${ }^{e}$ siècle, la littérature anglaise en anglo-normand offre tout un groupe de romans lignagers qui célèbrent chacun une grande famille aristocratique, le titre qu'elle porte et la région qu'elle contrôle : le Roman de Horn, Beuve de Hantone, Waldef, Gui de Warewic, Fitz Fouke Waryn glorifient des ancêtres fondateurs prestigieux, qu'ils hissent au rang de héros nationaux anglais, sans néanmoins les attirer du côté de la féerie ${ }^{8}$. En revanche, sur le continent, la vogue de la généalogie n’a encore donné naissance qu'à très peu d'œuvres en romanz qui relateraient le passé mythique d'une grande famille aristocratique : le roman Partonopeus de Blois, que son auteur présente comme une histoire des origines des comtes de Blois au temps du fils de Clovis, en est 
l'exemple majeur pour le XII ${ }^{\mathrm{e}}$ siècle, avant qu'au début du XIII ${ }^{e}$ siècle, la légende du Chevalier au Cygne ne soit retracée dans plusieurs œuvres, au bénéfice du lignage de Bouillon.

4 Or, exception faite du lai de Marie de France, ces œuvres en langue vernaculaire s'affirment presque toujours comme des textes hybrides, aux frontières de la chanson de geste, du roman et de la chronique, par leur vision du monde et des exploits héroïques - des aventures courtoises et/ou féeriques, entrelacées à des scènes guerrières, et notamment à de nombreux combats contre des Sarrasins - ainsi que par leurs procédés d'écriture. Les auteurs des premiers romans lignagers anglo-normands, Horn et Beuve de Hantone, adoptent d'ailleurs la facture des chansons de geste, avant que leurs successeurs ne lui préfèrent la succession continue des octosyllabes, pour rivaliser davantage avec les modèles d'écriture des romanz, et particulièrement des chroniques. De même, les poèmes qui constituent une ouverture aux chansons de la croisade, les deux versions de la Naissance du Chevalier au Cygne, le Chevalier au Cygne et la Fin d'Elias, sont composés en laisses épiques, alors que leur représentation de la féerie les rattache d'abord au genre romanesque et que la première version en français de la naissance du Chevalier au Cygne est celle d'un roman, le Dolopathos. Contrairement aux anglo-normands Horn et Beuve de Hantone, le découpage du récit en laisses épiques est peut-être moins un choix qu'une contrainte imposée par les liens établis avec les épopées de la croisade et la poétique de la continuation. Néanmoins, ces œuvres reflètent un croisement concerté des influences romanesque et épique, dont les thématiques ne cessent de s'entrelacer pour concourir à l'invention d'une mémoire prestigieuse du lignage.

5 Leur récit des origines a une double fonction de célébration et d'explication : il doit glorifier l'ancêtre qui transmet à ses descendants l'aptitude à conquérir Jérusalem et montrer pourquoi le lignage de Bouillon était prédestiné à réussir la croisade. Tout se passe en effet comme si la mission de croisade se voyait assignée à un lignage particulier, "captée » par ce lignage, célébrée à son profit, ce qui renforce les apparences de chronique familiale que prennent les poèmes en laisses épiques, bien qu'on ignore tout de leurs conditions précises d'écriture et de leurs mécènes éventuels $\mathrm{au} \mathrm{XIII}{ }^{\mathrm{e}}$ siècle. On remarque enfin que les poèmes versifiés du XIII ${ }^{\mathrm{e}}$ siècle et leur première mise en prose du XIII ${ }^{e}$ siècle ${ }^{9}$ datent d'une époque où le lignage a perdu le contrôle en Orient. S'agit-il de réaffirmer ses droits? De prouver que ses descendants peuvent encore réussir? On peut le supposer, et des motivations comparables expliquent encore, selon toute vraisemblance, les réécritures de ces récits d'origine à la fin du Moyen Âge, pour le bénéfice d'héritiers réels ou supposés des Bouillon. N'oublions pas non plus qu'au XIII ${ }^{\text {e }}$ siècle également, une œuvre d'inspiration très différente, le roman d'aventures Sone de Nansay, imagine une nouvelle "préhistoire " romanesque à la première Croisade, en remontant plus haut dans le temps et en inventant un ancêtre du Chevalier au Cygne, Sone de Nansay, qui serait le père de Matabrune, la grand-mère d'Elias. Le prologue en prose explicite ces liens généalogiques, lorsque l'auteur se présente comme le secrétaire d'une dame de Beyrouth, qui lui commande un récit généalogique pour que la célébration des exploits de son aïeul, Sone, incite les hommes de son époque à la croisade ${ }^{10}$.

6 À la fin du Moyen Âge et même au-delà, la légende du Chevalier au Cygne jouit toujours d'un grand succès en langue française et on connaît par ailleurs son extraordinaire diffusion dans la littérature germanique, qui, dès le XII ${ }^{e}$ siècle, l'associe à celle du Graal, 
avec l'établissement d'un lien de filiation direct entre Lohengrin et Perceval ${ }^{11}$. Le développement d'un «mythe de la croisade » à partir du XIV e siècle, si bien étudié par A. Dupront ${ }^{12}$, explique la floraison d'innombrables œuvres en français qui évoquent les expéditions des Croisés ou relatent des guerres fictives entre chrétiens et musulmans. Deux des adaptations tardives du premier cycle de la croisade nous permettront ici d'étudier comment leurs auteurs s'approprient, exploitent et transforment le récit des origines féeriques du Chevalier au Cygne et de sa destinée : la Chanson du Chevalier au Cygne et de Godefroy de Bouillon, longue ouverture du second cycle de la croisade, qu'on date de la première moitié du XIV ${ }^{\mathrm{e}}$ siècle, puis la mise en prose que Berthault de Villebresme compose au XVe siècle ${ }^{13}$.

7 L'auteur de la Chanson réécrit les poèmes du premier cycle de la croisade en posant de nouveaux accords entre le romanesque et l'épique. Il nous offre un bel exemple d'une "modernisation », dans la langue et dans l'inspiration, qui ne s'accompagne pas d'un changement de forme littéraire, puisqu'il conserve les laisses épiques. Si l'on date la rédaction de Bruxelles de 1356, la critique estime que le poème remonte aux années 1320-1330, quand, au début du règne de Philippe VI, les projets de croisade refleurissent : il s'agit alors de remémorer des exploits glorieux et susceptibles de faire renaître l'espoir dans le cœur de ceux qui voudraient répéter la " geste » de Godefroy. Les conditions de la rédaction de la mise en prose sont un peu mieux connues, notamment à travers la préface de l'auteur :

Duquel vouloir et naturel desir esprinse et louablement affectueuse, tres haulte, tres excellante et tres puissante princesse, ma tres redoubtee et naturelle dame, madame Marie de Cleves, duchesse d'Orleans, fille de prince de bonne et commendable memoire, monseigneur le duc de Cleves, a present veufve de feu prince de florissant et excellante renommee, monseigneur Charles, en son vivant duc d'Orleans, de Milan et de Valois, conte de Blois, de Pavye et de Beaumont, seigneur d'Ast et de Coucy, advertie et deuement informee que, par diverses generacions et successives dessentes de ligne en ligne, elle et ses predecesseurs, ducs et seigneurs de Clevez, sont issus, partiz et dessenduz d'un tres noble et tres victorieulx chevallier, filz de roy, nommé Helyas - et par merveilleuse aventure cy après descripte et recitee, denommé le chevallier au cigne- desirant icelle dame de tout son cueur savoir et entendre la merveilleuse naissance, l'estrange adventure et les triumphans victoires dudict chevallier et de sa tres noble et historieuse posterité et lignee, contenue en ung livre a elle, nagueres envoyay en ancienne rime et assez obscur lengaige dificille a compregnoir et entendre, ait comandé a moy, Berthault de Villebresme, son tres humble subgect et indigne serviteur, mectre et translater icellui livre et memoriable histoire de ladicte ancienne rime et obscure lengaige en prose et lengaige françois cler et entendible, ad ce qu'elle et les liseurs et escouteurs d'icellui puissent plus facillement avoir congnoissance de la merveilleuse et plaisent histoire dudict chevallier et de son tres noble et glorieulx lignaige.

Berthault de Villebresme, qui a vécu à la cour de Charles d'Orléans et participé à son concours de ballades sur le thème "Je meurs de soif aupres de la fontaine ", la réalise donc pour sa veuve, Marie de Clèves, vers 1470 . On sait que la famille de Clèves, après l'extinction du lignage de Boulogne-Bouillon, s'est effectivement approprié le Chevalier au Cygne comme ancêtre et rêvait de reprendre le flambeau de la croisade, pour donner à la chrétienté un nouveau Godefroy ${ }^{14}$. Alphonse de Clèves, le père de Marie de Clèves, s'était engagé à partir pour la croisade au fameux banquet du Faisan de Lille. Selon Olivier de la Marche, la journée avait commencé par une joute qu'il avait annoncée, en promettant au vainqueur un riche cygne d'or : 
Tel fut le cry, par l'ordonnance et adveu de monseigneur Adolf de Cleves, lequel estoit celluy pour qui la criée se faisoit, et, a ce que je veiz, la criée et jouste se faisoit au propos d'ung entremetz, qui contenoit, a ce dit bancquet, la pluspart de la longueur de la principalle table. Ce fut une nef a voille levée, moult bien faicte, en laquelle avoit un chevalier tout droit, armé, qui le corps avoit vestu d'une coste d'armes, des plaines armes de Cleves, et devant avoit ung cigne d'argent portant en son col ung collier d'or auquel tenoit une longue chaine d'or dont ledit signe faisoit maniere de tirer la nef, et au bout de ladicte nef seoit ung chastel moult bien faict et richement, au pied duquel flotoit ung faulcon en une grosse riviere. Et me fut dit que ce signiffioit et monstroit, comme jadiz miraculeusement ung signe amena dedans une nef, par la riviere du Rin, ung chevalier au chasteau de Cleves, lequel fut moult vertueulx et vaillant, et l'epousa la princesse du pays, qui pour lors estoit vefve et en eut lignée, dont lesditz ducs de Cleves, jusques a ce jour, sont yssuz ${ }^{15}$.

Voici ensuite comment ce nouveau «Chevalier au Cygne » met en scène son arrivée au banquet :

Monseigneur Adolf [...] partit de son hostel a grant compaignie de gens vestuz de ses robes, et alloient devant; et apres eulx alloient tabourins, et apres alloit un poursuyvant d'armes, vestu d'une cotte d'armes pleine de signes, et apres alloit ung grant signe merveilleusement et subtilement faict, ayant une couronne d'or au col, a quoy pendoit ung escu des plaines armes de cleves, et a celle couronne pendoit une chaisne d'or, qui d'ung bout tenoit a la tresse de l'escu du chevalier, et estoit ce signe adextré de deux sagittaires moult bien faictz, qui tenoient arcs et fleches en leurs mains, et faisoient semblant de tirer a l'encontre de ceulx qui voulloient aprocher le cigne. Ledit chevalier, tenant a la chaisne d'or, suyvoit le signe, armé tres richement de toutes armes ${ }^{16}$.

Le second entremets du banquet représente plus loin « ung chasteau a la façon de Lusignian, et sur ce chasteau, au plus hault de la maistresse tour, estoit Melusine en forme de serpente $»^{17}$.

11 Les légendes de Mélusine et du Chevalier au Cygne circulent alors parallèlement, bien que celle du Chevalier au Cygne connaisse une plus grande diffusion à la cour de Bourgogne. Toutes deux tissent des liens mystérieux entre la féerie, le surnaturel chrétien et la mission de croisade. Les Romans de Mélusine, qui datent de la fin du $\mathrm{XIV}^{\mathrm{e}}$ siècle et du début $\mathrm{du} \mathrm{XV}^{\mathrm{e}}$ siècle, sont commandés par des mécènes qui ne semblent pas avoir rêvé à la croisade mais qui, dans le contexte de la guerre de Cent Ans, cherchent à s'imposer comme les descendants de la fée pour revendiquer la possession de Lusignan et du Poitou. Non seulement Jean d'Arras et Coudrette ne profitent pas de leur liberté de créateurs de fiction pour projeter dans le passé des origines une prise mythique de Jérusalem, mais ils referment les espoirs de reconquête avec l'expédition de Geoffroy à la Grande Dent et l'aventure manquée de Palestine ${ }^{18}$. Dans les Vœux $d u$ Faisan d'Olivier de la Marche, l'évocation de Mélusine reste d'ailleurs très brève et la fée n'est pas rattachée à un lignage précis. Avec le Chevalier au Cygne, ancêtre présumé des Bouillon et des Clèves, la commémoration du passé semble au contraire ouvrir un nouvel avenir, comme si l'œuvre de mémoire allait donner prise sur le réel. Vers 1500, le souvenir des premiers Croisés éveille toujours l'intérêt, comme l'atteste la composition par Pierre Desrey de la Genealogie de Godefroy de Bouillon: «s'adressant à Louis XII et Engelbert de Clèves, il veut leur conter l'histoire des ancêtres qui ont combattu pour la défense de la foi, le roi de France peut se reconnaître en Louis VII, 
Philippe-Auguste et surtout saint Louis, et Engelbert de Clèves dans le Chevalier au Cygne, puisque telle était, au $\mathrm{XV}^{\mathrm{e}}$ siècle, la revendication généalogique de cette maison $»{ }^{19}$

La Chanson du Chevalier au Cygne et de Godefroy de Bouillon et la mise en prose de Berthault de Villebresme restent fidèles à la trame narrative générale de la Naissance du Chevalier au Cygne, du Chevalier au Cygne et de la Fin d'Elias. Aucune de ces deux adaptations n'introduit une innovation aussi déterminante que le rattachement au mythe du Graal dans les œuvres germaniques. Un seul auteur français, celui de Sone de Nansay, a cherché à souder, au XIII ${ }^{\mathrm{e}}$ siècle, les deux mythes d'origine, le mythe du Graal et le mythe du Chevalier au Cygne, en célébrant son héros comme le fils spirituel de Joseph d'Arimathie et en lui accordant le privilège d'une visite au château du Graal, qu'il imagine en Norvège. L'auteur de la Chanson du Chevalier au Cygne réécrit néanmoins entièrement les poèmes dont il s'inspire, comme si le choix du même vers, l'alexandrin, et d'une même structure strophique, le contraignait d'autant plus à un renouvellement et à une réinterprétation de la légende. Son prologue, qui annonce l'ensemble des hauts-faits que célébrait le premier cycle de la croisade, n'apporte aucune explication sur les rapports que son texte entretient avec ses sources. Le projet de réunir dans un même récit, d'unifier à travers une même écriture et un même point de vue, des œuvres composées par des auteurs différents, à des dates différentes, puis juxtaposées par plusieurs copistes dans l'espace d'un même manuscrit, n'est donc jamais exposé. Renonçant pourtant à la pratique de la continuation qui conduisait ses prédécesseurs à toujours ajouter de nouveaux textes, l'auteur préfère prolonger la mise en cycle, imputable jusqu'alors aux seuls copistes, en un travail d'adaptation et de recréation. L'aboutissement en est la constitution d'une seule et même œuvre, le tissage d'un nouveau texte, plus continu, plus homogène et cohérent, qui est destiné à remplacer les poèmes du premier cycle. Tout se passe alors comme s'il anticipait sur les objectifs qui seront ceux de nombreux auteurs de mises en prose du $\mathrm{XV}^{\mathrm{e}}$ siècle, mises en prose de chansons de geste, de romans ou de textes hybrides comme les Romans d'Alexandre: pensons à Jean Wauquelin pour la réécriture de l'immense cycle épico-romanesque sur Alexandre le Grand, lui qui « convertit » en une œuvre unique la multiplicité des textes versifiés composés du XII ${ }^{e}$ au XIV ${ }^{\mathrm{e}}$ siècle autour du Roman d'Alexandre d'Alexandre de Paris. Mais au XII ${ }^{e}$ siècle, dans la facture des laisses épiques, Alexandre de Paris avait déjà mené à bien un travail comparable d'assemblage et de refonte de récits français antérieurs. Dans la Chanson du Chevalier au Cygne, la modernisation de la langue, du vocabulaire et des techniques du récit, quoique non revendiquée comme elle l'est souvent dans les mises en prose $\mathrm{du} \mathrm{XV}^{\mathrm{e}}$ siècle, s'avère très aboutie, en dépit même de la conservation des laisses épiques, concession apparente aux modes du passé : elle repose ainsi sur le paradoxe apparent qui est de choisir une forme ancienne, vieillie, pour innover. La mise en prose apparaît en revanche moins originale, car elle ne reflète pas une réinterprétation de l'histoire du Chevalier au Cygne.

L'analyse et la comparaison de leurs adaptations de la Naissance du Chevalier au Cygne est d'autant plus intéressante qu'il existait de ce poème deux versions du XIII siècle, désignées par les éditeurs du nom de la mère d'Elias dans chacune d'entre elles, Elioxe et Beatrix. L'auteur de la Chanson du Chevalier au Cygne et de Godefroy de Bouillon choisit de les concilier tout en les modifiant très sensiblement, ce qui prouve bien sûr qu'il les connaissait toutes les deux, mais surtout qu'il prend parti, que son adaptation est le fruit d'une lecture attentive des textes et d'un examen de leurs enjeux. Cette analyse le conduit à reconsidérer la signification de nombreux épisodes, à sélectionner, omettre 
ou ajouter, et elle aboutit à un infléchissement sensible de l'image des origines surhumaines d'Elias. Un assemblage des deux récits avait déjà été réalisé dans une version du XIII siècle dite "composite", mais son auteur se contentait alors de réécrire fidèlement le début d'Elioxe - le récit de la rencontre amoureuse et du mariage $\mathrm{du}$ père d'Elias avec une créature féerique - pour ensuite reprendre tout aussi précisément Beatrix : plutôt que de faire mourir la mère des enfants-cygnes en couches comme dans Elioxe, il relatait le martyr que sa belle-mère lui inflige et la confrontation violente d'Elias avec sa grand-mère Matabrune dont il avait lu le récit dans Beatrix ${ }^{20}$. L'auteur de la Chanson s'accorde en revanche de grandes libertés, tant et si bien qu'en dépit d'une fidélité aux grandes lignes de l'intrigue, son récit des origines acquiert une signification nouvelle. Cette dernière semble alors témoigner d'une réflexion sur les ascendances maternelle et paternelle qu'il convient de prêter à l'ancêtre de Godefroy de Bouillon, car elles déterminent son statut ontologique, ainsi que sur les exploits à lui attribuer pour annoncer et expliquer la glorieuse carrière du héros de la première Croisade. Quant au prosateur $\mathrm{du} \mathrm{XV}^{\mathrm{e}}$ siècle, comme l'auteur de la première mise en prose du XIII siècle, il se limite en revanche à suivre beaucoup plus fidèlement Beatrix.

D'après le nombre de manuscrits conservés des deux versions de la Naissance $d u$ Chevalier au Cygne et aussi d'après ces mises en prose, c'est en effet Beatrix qui a joui du plus large et plus durable succès. La critique en a donné pour explications la mise en avant du personnage d'Elias, au détriment de ses frères et de sa sœur, le personnage principal d'Elioxe, et la christianisation très insistante du Chevalier au Cygne. Les nouveaux exploits d'élu de Dieu qui lui sont attribués permettent en effet de rattacher plus étroitement Beatrix aux autres poèmes du cycle, en annonçant la croisade. Dans Elioxe et dans le Dolopathos, c'est au contraire la sœur des six fils de la fée qui échappe à la métamorphose en cygne et œuvre ensuite au triomphe de la vérité, parvenant à innocenter sa mère et à redonner à ses frères, à l'exception de l'un d'entre eux, leur forme humaine. Elioxe se clôt alors sur le départ des cinq frères, qui s'orientent dans des espaces différents, si bien que cette « diaspora » du lignage semble préfigurer une main mise sur l'univers tout entier. Le poème qui suit dans le cycle, bien que sa date de composition soit peut-être antérieure, le Chevalier au Cygne, n'évoque néanmoins jamais la destinée des quatre frères d'Elias, car le récit généalogique se consacre exclusivement à l'ancêtre direct de Godefroy. La continuation "par l'avant " que constitue Elioxe ouvrait ainsi des perspectives narratives très foisonnantes et très romanesques, puisque des aventures diverses sont proposées à chacun des frères. Pour les deux premiers, ce sont des aventures qui rappellent les récits bretons par les espaces féeriques qu'elles privilégient, la noire montaigne (v. 3337) avec son bestiaire exotique, la forêt qui permet de s'esprover (v. 3345). Le troisième décide de s'illustrer dans un royaume de femmes guerrières et l'évocation explicite des Amazones, la perspective d'une conquête de leur pays et d'une idylle avec leur reine, tissent des liens d'intertextualité évidents avec les romans d'Antiquité et notamment les Romans d'Alexandre (v. 3352-3359). Le quatrième élit ensuite la mer et une aventure bien déterminée, celle de la montagne d'aimant, qui, issue de contes orientaux, est exploitée dans plusieurs récits d'aventures du XIII ${ }^{\mathrm{e}}$ siècle, tant romanesques qu'épiques (v. 3360-3366). Seul le Chevalier au Cygne refuse de choisir son destin. Il préfère s'en remettre à la volonté divine, tout en s'assignant le devoir de veiller sur son frère-cygne, sans que l'auteur reprenne l'annonce de la croisade, qui marque pourtant le début de son texte, ni qu'il réaffirme une inspiration épique qui permettrait de mieux souder son œuvre aux suivantes. 

florilège de textes, qui aurait pu constituer une arborescence autour du tronc commun sur le Chevalier au Cygne. L'auteur précise lui-même qu'il s'apprête à sélectionner dans cette riche « matière », pour restreindre son champ narratif à Elias et au cygne, comme s'il était l'auteur du Chevalier au Cygne. Son invitation implicite à l'écriture sur les autres frères est d'ailleurs restée lettre morte, tant au XIII siècle qu'à la fin du Moyen Âge, et son concurrent, l'auteur de Beatrix, a supprimé ces fils narratifs annexes en réorganisant l'ensemble du récit autour d'Elias. La relégation de la sœur au rôle de personnage secondaire conforte le discours misogyne violent qui parcourt l'ensemble de Beatrix et surtout permet de célébrer Elias, dont l'exemplarité passe par une accumulation de marques nouvelles de son élection divine: miracles, interventions d'anges, de troupes célestes, duel judiciaire victorieux. Le surnaturel féerique se voit complètement écarté au profit du surnaturel divin, la christianisation contribuant très efficacement à expliquer l'élection des petits-fils pour la croisade. Ainsi disparaît le lien énigmatique qui unit la féerie à la croisade dans Elioxe. Un dernier point est à noter, sans doute aussi très important : dans Elioxe, le futur chevalier au cygne subit, avec ses frères, la métamorphose animale. Beatrix lui épargne cette muance, qui, selon la légende, est toujours synonyme de souffrance et qui en outre, dans les esprits médiévaux, soulève implicitement la question de la création de l'homme à l'image de $\mathrm{Dieu}^{21}$. Au moment de la transgression de l'interdit par la duchesse de Bouillon dans le Chevalier au Cygne, Elias disparaît d'ailleurs aussi sans se départir de son apparence humaine. Il est alors indéniable que dans Beatrix la focalisation sur Elias et la réinvention de son portrait - de l'enfant-cygne impuissant au chevalier de Dieu rayonnant et invincible - établissent une meilleure continuité avec le Chevalier au Cygne.

$\mathrm{Au}$ XIV e siècle, l'auteur de la Chanson du Chevalier au Cygne et de Godefroy de Bouillon choisit néanmoins de s'inspirer d'Elioxe pour commencer le récit des origines du lignage et nourrir le portrait des parents d'Elias. S'il renoue avec la version de Beatrix juste après l'épisode du mariage, c'est en lui apportant de sensibles modifications. Son adaptation traduit donc un «croisement » des deux œuvres dont les enjeux restent à définir. Comment expliquer tout d'abord le retour à Elioxe, qui est censé fourni un meilleur point de départ? Rappelons que Beatrix commence très abruptement par la calomnie de l'épouse du roi Oriant, qui, voyant une mère accompagnée de ses jumeaux, met en cause méchamment sa vertu, avant d'être «punie " par le plaisir de Dieu, le Pere omnipotent (v.77), en concevant des septuplés. L'auteur de Beatrix efface ainsi la séquence de la première rencontre des futurs époux, qui révélait l'appartenance féerique du personnage maternel dans Elioxe et encore plus clairement dans le Dolopathos. Les naissances multiples, que de nombreuses légendes introduisent comme un signe de féerie, sont ici réinterprétées en relation avec la faiblesse maternelle et la volonté divine. Au-delà du silence sur ses origines, la mère apparaît désormais humaine, trop humaine, par la faute qu'elle commet d'accuser injustement d'adultère une autre femme. La parole maternelle déclenche le récit, mais loin d'être une parole prophétique, qui trahirait le savoir supérieur dont disposent les fées, elle engendre le malheur, en signifiant son ignorance et son manque de charité, que sa belle-mère a beau jeu d'exploiter. Pensons alors, dans le Lai de Fresne de Marie de France et dans son adaptation romanesque Galeran de Bretagne, au portrait de la "mauvaise " mère de Fresne, qui prononce la même accusation avant d'être aussitôt châtiée. Le regard très négatif qui pèse sur la femme tout au long de Beatrix n'épargne donc pas la propre mère du Chevalier au Cygne, même si l'auteur noircit ensuite bien davantage Matabrune, la 
grand-mère. Le poème intitulé le Chevalier au Cygne prolonge cette dénonciation des «faiblesses » féminines. Certes, c'est alors le motif de l'interdit imposé par l'être faé qui suppose la transgression et donc une condamnation de la curiosité et de la trahison de la duchesse de Bouillon. Malgré la douleur qu'elle affecte au départ de son époux, l'épouse confirme cependant sa déloyauté, car elle oublie rapidement la dernière mission qu'il lui a confiée, la garde du cor merveilleux, ce qui provoque la destruction du château de Bouillon. Seule la fille du Chevalier au Cygne est finalement épargnée, mais elle est valorisée moins pour ses qualités personnelles que pour son rôle de génitrice des héros de la première Croisade. Voici l'un des paradoxes de ce récit des origines : il concilie une promotion de plusieurs femmes comme maillons essentiels du lignage et un discrédit jeté sur leurs "péchés", les valeurs courtoises restant étrangères à l'auteur.

La Chanson du Chevalier au Cygne et de Godefroy de Bouillon, sans supprimer cette condamnation maternelle des naissances multiples, lui dénie l'importance qu'elle prend dans Beatrix. Ainsi n'est-elle plus mise en avant, au début de la narration, comme ce qui enclenche l'engrenage de la violence. L'auteur préfère revenir au point de départ d'Elioxe, un récit de la rencontre du père et de la mère d'Elias qui s'inspire des scénarios féeriques de la découverte d'une fée par un chevalier mortel. L'effacement de l'ascendance féerique maternelle dans Beatrix se conciliait en effet difficilement avec la résurgence de la féerie à travers l'interdit mélusinien dans le Chevalier au Cygne. Dans un souci probable de cohérence, il lui a donc semblé nécessaire de suggérer à nouveau que la nature féerique du Chevalier au Cygne lui venait de sa mère, ainsi que d'estomper la «méchanceté » qui ternissait la première image de la figure maternelle. Les sept enfants sont ainsi conçus avant même la mise en cause de la mère des jumeaux, le jour même du mariage d'Oriant et de Beatrix (v. 185-225), alors que dans Beatrix (1. 5), cette conception succédait à la calomnie, tel un châtiment, comme c'est aussi le cas dans le Lai de Fresne de Marie de France. La modification de l'ordre des événements semble alors signifier la gêne de l'auteur devant ce lien possible de cause à effet et cette explication, étrange et peu glorieuse pour les descendants du lignage, des naissances multiples.

18 La réintroduction de la féerie connait néanmoins des limites très nettes. Comme dans Elioxe, une différence sépare la mère de ses fils : la fée n'apparait plus sous les traits d'une femme-cygne, que le chevalier découvrirait au bain et dont il déroberait la robe de plumes ou la chaîne merveilleuse comme dans le Dolopathos. La possibilité d'une métamorphose en cygne n'est jamais explicitée comme un héritage de l'ascendance maternelle, mais interprétée comme un don de Dieu. En effet, la christianisation de la légende n'est pas l'apanage de Beatrix au XIII ${ }^{\mathrm{e}}$ siècle, elle est déjà bien effective dans Elioxe, où la féerie semble étroitement contrôlée par la volonté divine. Or, ce contrôle divin se renforce encore dans l'adaptation du $\mathrm{XIV}^{\mathrm{e}}$ siècle, comme l'attestent une rationalisation plus profonde du merveilleux et l'effacement de deux épisodes importants d'Elioxe : le discours prophétique que la jeune femme adresse au roi, le jour de leur première rencontre (v. 249-274), et le récit de son rêve prémonitoire (v. 468-525).

19 La Chanson du XIV ${ }^{e}$ siècle abrège et simplifie l'épisode initial de la rencontre: la demoiselle est découverte au terme d'une chasse à l'animal sauvage - ung grant cerf-, lorsque le chevalier s'arrête près d'une fontaine pour s'y rafraîchir. La suppression de nombreux détails descriptifs efface plusieurs des signes évidents de la féerie - le 
caractère androgyne de l'animal merveilleux par exemple -, mais la rationalisation apparente n'empêche pas de reconnaître le scénario féerique traditionnel, qui, dans la littérature médiévale, n'a jamais besoin d'être parfaitement explicité pour être perçu comme tel. De surcroît, alors qu'Elioxe séparait le moment de repos du chevalier à la fontaine de celui de la rencontre féerique, l'auteur du XIVe siècle choisit de les faire à nouveau coïncider, comme s'il s'agissait de renouer avec l'un des signes les plus conventionnels de la féerie :

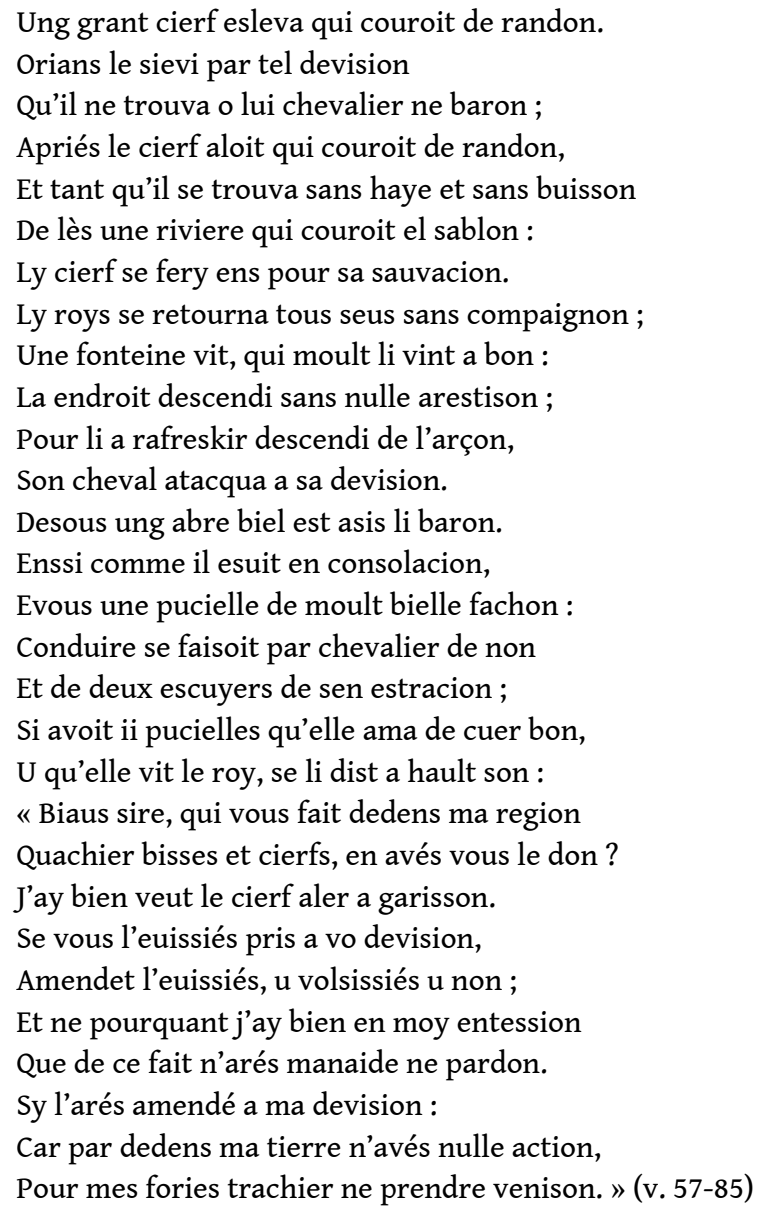

L'énigme qui entoure les origines de la jeune femme n'est jamais élucidée. Qui plus est, elle ne suscite aucune interrogation de la part du héros ou du narrateur. Contrairement à son fils et à d'autres créatures mélusiniennes, Beatrix n'impose d'ailleurs jamais aucun interdit, si bien qu'à son sujet la question sur les origines disparaît en tant que tabou. À l'instar de nombreuses fées, elle se présente comme une dame féodale, soucieuse de ses droits, avant de s'incliner devant Oriant, lorsqu'il la replace sous son autorité de suzerain et de futur époux. Le rapport de force bascule ainsi très vite en faveur du roi. Le chevalier qui accompagne la dame intervient pour l'excuser comme si elle n'était qu'une jeune étourdie, avant que le silence ne relègue dans l'oubli le monde auquel elle appartient. La féerie est ainsi réintroduite sur le simple mode de la suggestion, grâce à une écriture qui sollicite la mémoire des auditeurs-lecteurs et leur connaissance des légendes féeriques, une écriture qui affecte la transparence tout en éveillant le mystère. La jeune femme, étrangère plus qu'étrange, s'impose par sa beauté et l'amour immédiat qu'elle éveille, non par ses richesses ou son intelligence politique $^{22}$. Elle n'apporte ni la prospérité ni la puissance, comme le fera la fée Mélusine dans les romans de la fin du Moyen Âge, mais sa fécondité extraordinaire assure la 
naissance d'un lignage glorieux. Après le long exil que lui impose Matabrune à l'intérieur même de la cour d'Oriant, elle réussit à s'intégrer durablement dans le royaume de son époux, puisque l'auteur lui épargne une mort prématurée ou un départ contraint et lui confère alors une pleine nature de mortelle.

La parole prophétique que la fée d'Elioxe adresse au roi le jour même de leur rencontre disparaît également de l'adaptation du XIVe siècle. L'héroïne d'Elioxe annonçait que leurs descendants étaient prédestinés à régner sur la Terre sainte et qu'ils naîtraient avec une chaîne en or. Elle ajoutait qu'elle était condamnée à mourir en couches :

Se me prendés a femme, por voir vos puis conter,

Vostre linages ert espandus outre mer

Et jusqu'en Oriant le verra on regner.

[...] En la premiere nuit aprés nostre espouser,

Que vauras vraiement a ma car deliter,

Jo te di par verté loiaument sans fauser

Que tu de.VII. enfans me feras encarger :

Li .VI. en ierent malle, et pucele al vis cler

Iert li sietismes enfes, co ne puet trespasser.

Lasse! Moi, j'en morrai de ces enfans porter.

Et quels talens me prent que jo m'en doie aller

La u il m'estavra de tele mort pener,

Mais que teux destinee doit par mi moi passer?

Et m'estuet travellier et tel mort endurer

Por le linage acroistre qui ira outre mer,

Et qui la se fera segnor et roi clamer.

Encore te vaurai jo autre cose conter :

Cascuns de ces enfans aura sisne d'or cler

El col d'une caaine que bien porra mostrer ;

A tot co naistront il jel vos di sans doter.

Commandés les enfans par grant cure a garder,

Quel part que vos soiés, $u$ en terre $u$ en mer

Ou en pais ou en guerre, vos en estuet pener. (Elioxe, v. 250-274)

Des liens mystérieux existent donc entre la féerie et la croisade, comme si la réussite de la croisade devait absolument être prédite par une voix féerique et réalisée par des héros qui appartiendraient à l'Autre monde du fait de leur ascendance maternelle. La créature féerique, même si le terme de "fée » n'est pas employé, connaît l'avenir. Ce savoir prophétique n'est pas synonyme de toute-puissance, car elle se sait vouée à un départ définitif - la mort et non un retour dans l'Autre Monde -, sans qu'il soit question de la transgression d'un interdit par son époux. Elle apparaît finalement comme une envoyée de Dieu, dont la mission est d'engendrer un lignage qui se consacrera à la croisade. Remarquons aussi qu'Elioxe ne révèle pas tout à son époux. Elle occulte les vertus merveilleuses des chaînes et la métamorphose des enfants en cygnes, bien que le jeu de mots sur sisne (v. 269), de même que la mise en garde sur la nécessité de bien protéger les enfants, laisse entendre qu'elle tait ce qu'elle sait.

Dans le poème du second cycle de la croisade, ce don de divination lui est retiré. L'auteur préfère se l'approprier symboliquement, avec le procédé épique de l'annonce en fin de laisse. Une prolepse du narrateur remplace ainsi le discours de la fée :

Elaes! que ceste amour fu bien tos departie

Par la mere du roy, dont elle fu haïe,

Matabrune, qui puis en fu arse et bruie

Par le boin Helyas et sa chevalerie.

Le Chevalier au Chine, a le chiere hardie, 
Vint de ceste rachine et de cheste lingnie,

Godefroy de Buillon qui conquesta Surie,

Witasse et Bauduin qui tant ot de seignourie,

Ensi que vous orés en l'istore jolie. (v. 134-142) qu'elle reçoit dans Elioxe, sans d'ailleurs le comprendre clairement. Tout se passe comme si l'auteur de l'épopée romanesque du $\mathrm{XIV}^{\mathrm{e}}$ siècle souhaitait donner une meilleure transparence à son récit, supprimer les mystères non résolus. Le songe ne se contentait pas d'annoncer les malheurs à venir, mais avec l'image de la pomme du Paradis et le rappel du péché originel, il ouvrait le récit sur une profondeur romanesque, il laissait la voie libre aux interprétations, sollicitait le lecteur, l'engageait dans une quête de sens.

L'adaptation du $\mathrm{XIV}^{\mathrm{e}}$ siècle, qui semble conçue pour livrer une signification plus univoque, efface donc ce qui donnait à la fée d'Elioxe un caractère à la fois sublime et mystérieux : son savoir surnaturel, son acceptation du sacrifice, la relation privilégiée avec Dieu qu'ils révèlent. Comme son auteur s'inspire de Beatrix à partir de l'épisode du mariage, il lui était certes impossible de laisser mourir en couches son héroïne, mais rien ne le contraignait à abréger autant. Dans Elioxe, en dépit de sa disparition rapide, la fée apparaît comme la véritable héroïne et la première ancêtre du lignage, consciente de son devoir et de sa destinée, et ce n'est sans doute pas un hasard si c'est ensuite une autre figure féminine, sa fille, qui passe au premier plan et se dévoue pour sauver ses frères et permettre au lignage d'accomplir son destin. En limitant le rôle de la fée et de sa fille, l'auteur du XIV siècle redonne à Elias sa place de vrai fondateur du lignage, tout en supprimant les liens problématiques entre la féerie et la croisade, entre la créature féerique et Dieu, qu'Elioxe tissait. Dans la Chanson, au-delà de la prolepse du narrateur, la parole prophétique est désormais déléguée à l'ermite, qui a reçu ces révélations d'un ange (v. 1165-1169). La réussite de la croisade n'a plus besoin du relais de la parole de la fée qui, à partir du moment où elle subit les accusations de sa bellemère, semble perdre son statut d'être féerique et, comme dans Beatrix, l'élection d'Elias par Dieu le métamorphose finalement en une créature plus divine que féerique.

Pour le portrait de la figure maternelle persécutée, l'adaptation du XIV ${ }^{\mathrm{e}}$ siècle se caractérise alors avant tout par un renforcement du pathétique. Non seulement la jeune femme se repent immédiatement de sa parole calomnieuse, qui n'entraîne aucun châtiment, mais un accent nouveau porte sur les manifestations de sa souffrance. La nature des tourments qu'elle endure change, car la violence physique et les atteintes corporelles, qui dominent le Dolopathos et Beatrix, laissent la place aux souffrances psychiques. En dépit de son emprisonnement, elle ne subit plus de traitements dégradants et n'est plus contrainte de régresser à l'état de beste mue. Selon Beatrix en effet, la vieille Matabrune la bat, puis la livre à des serfs qui la brutalisent :

\footnotetext{
Li serf en ont menee la dame toute nue

Fors que d'une pelice dont ele estoit vestue.

En la cartre l'en mainent sans nule retenue ;

Drap ne cousin ne kiute n'a avoec li eüe,

.I. poi d'estrain li jetent con une beste mue. (Beatrix, v. 283-287)
}

Dans la Chanson, le roi Oriant ordonne au contraire qu'on traite dignement son épouse. Mais l'auteur invente une nouvelle source de souffrance morale, qui amplifie le pathos. $\mathrm{Si}$, comme dans Beatrix, après avoir substitué des chiots aux nourrissons, Matabrune l'accuse de relations sexuelles avec des chiens, les effets de la perversité de la belle- 
mère sont désormais décuplés par l'ignorance dans laquelle la jeune femme est placée. L'auteur du XIV siècle imagine en effet que les douleurs de l'accouchement l'empêchent de voir ses enfants et lui retirent donc la certitude de leur nature humaine: «le mal qu'elle senti a son cuer aveuglé ;/ne vit ne ne senti ce qu'el ot enfanté » (v. 324-325). Quand elle découvre les chiots, elle est alors dans l'incapacité de mettre en doute la parole de Matabrune :

«Pute, dist Matabrune, ne valés I bouton!

Digne estes de l'ardoir en un feu de carbon

Qui a ung chien avés eut amjonction!» (Chanson, v. 368-370)

d'autant que le mensonge de cette dernière est cautionné par la matrone, la sagefemme. Ce nouveau personnage assiste Matabrune et confère à la narration une plus grande vraisemblance. Le travail d'adaptation et de renouvellement s'accompagne toujours d'un souci nouveau pour la vraisemblance, d'une attention plus grande portée à la cohérence : de nouvelles précisions, ici un personnage supplémentaire, rendent plus crédible l'enchaînement des événements. Dans Beatrix, les manigances de Matabrune réussissaient en dépit de toute vraisemblance : l'absence du roi au moment de l'accouchement ne recevait aucune explication, sa naïveté et sa passivité semblaient étonnantes, à moins de prêter des pouvoirs surnaturels d'ensorceleuse à Matabrune, qui assistait seule à la naissance des enfants. Dans la Chanson du XIV ${ }^{\mathrm{e}}$ siècle, la jeune mère affronte désormais un duo infernal. Croyant avoir réellement enfanté des chiens, elle est submergée par un sentiment de culpabilité que Matabrune et sa complice exploitent avec habileté :

- « Lasse ! dist la royne, ja n'y aray pardon ;

Se ly roys Orians, qui tant a de regnon,

Siet ceste cose chy par nesune ocquoison,

Jamais n'aura a moy jour consolacion :

Car digne ne suy pas que j'aie audission,

Amour ne druerie d'omme ne de garçon,

Quant de moy est issue telle desolacion.

Digne suy de morir a grant destruction ;

Et au moins se li rois me donnoit I seul don,

D'aller et demourer en la religion

D'une povre abye, pour y estre en orison,

J'en loeroie Dieu qui souffry passion. » (v. 383-394)

Jamais la parole ne lui est retirée comme dans Beatrix. Les tourments lui permettent au contraire de prouver sa vertu et sa piété, mais la honte dans laquelle elle s'enferme l'empêche de se défendre. L'auteur prend donc soin de préserver sa dignité, comme en témoigne aussi la nouvelle prière qu'il lui accorde pour insister sur sa dévotion à la Vierge (v. 571-588) :

- Lasse! dist la royne, vechy mon corps trahy.

Très douce mere Dieu, de cuer vous ay siervi.

Mais par confait peciet pui ge avoir desiervi,

Que VII kiens sont issut enssi du corps de my?

Que m'est il avenu? je ne say que c'est chy!

Or ay pierdu l'amour de mon loiel amy,

De cheli qui m'amoit, et je l'amoye oussy

Dou plus biel, du meilleur c'oins de mere nasky. (v. 571-578)

Ce dégoût pour elle-même, pour son propre corps, elle le ressent jusqu'à ce qu'elle apprenne la vérité, de la bouche de son propre fils, et puisse en finir avec ce sentiment d'étrangeté. 
31 L'évolution de son personnage est alors indissociable du renouvellement des portraits $\mathrm{du}$ roi et de Matabrune. La liberté de l'adaptateur se manifeste ici à travers des retouches si nombreuses qu'elles conduisent presque à une métamorphose des deux personnages. Le roi Oriant, très épris de sa femme, exprime son désespoir lorsqu'il apprend les naissances monstrueuses. Si aucun doute ne l'assaille sur les paroles de sa mère, c'est qu'elles sont confortées par le témoignage de la sage-femme (v. 519-535). Tout en croyant aux relations sexuelles de son épouse avec un chien (jamais il n'émet l'hypothèse que cette descendance canine puisse être la sienne), il ne l'en accuse pas et ne manifeste aucun mouvement de répulsion. Seule la certitude de perdre son autorité de roi auprès de ses vassaux l'empêche de poursuivre la vie conjugale avec elle :

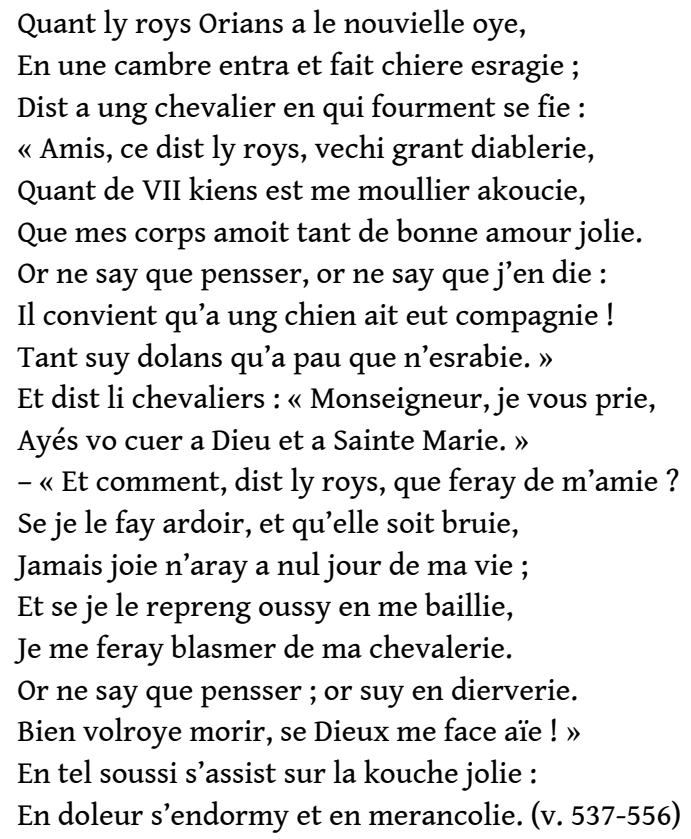

Alors que dans Beatrix son personnage insignifiant, totalement aliéné à Matabrune, offrait une piètre image paternelle, l'auteur de la Chanson s'emploie très nettement à le réhabiliter. Il l'affranchit de l'autorité de sa mère, lorsqu'il ajoute ensuite une scène qui n'a aucun équivalent dans Beatrix et reflète une modification complète du point de vue : le jugement que le roi organise pour décider du sort de son épouse.

Tandis que Matabrune régnait seule dans Beatrix et imposait soumission à tous par la terreur qu'elle inspirait, Oriant détient désormais le pouvoir et assume ses devoirs de roi. Face à ce «cas » étrange des naissances animales, il convoque ses contes et ses chevaliers, s'assure la présence d'un évêque et tient conseil. La scène est marquée par la précellence accordée à la parole de l'évêque. Or, cet homme de Dieu, cette figure de sagesse et d'autorité, innocente immédiatement la jeune femme en invoquant comme seule explication possible de la naissance des chiots le viol qu'un chien lui aurait fait subir :

Espoir qu'en son dormant, enssy que sommes prent,

Une bieste li ait fait ce vilain tourment,

Che ne fu pas son gret ne son consentement (v. 621-623).

Il ajoute aussi qu'en vertu du sacrement du mariage et en souvenir de leur amour, le roi commettrait une injustice en la condamnant à mort, tant et si bien qu'il l'exhorte à la protéger et à s'en remettre au jugement de Dieu : 
« ...faites la royne garder sougneusement

En un lieu gracieus, a vo commandement,

La u elle ait sa vie bien et souffisaument,

Et s'en laissiés a Dieu prendre l'avengement. » (v. 629-632) attaque païenne : son devoir politique et religieux le contraignait alors à quitter la cour pour s'illustrer dans une croisade avant la lettre. Cette séquence guerrière, longuement amplifiée, permettait de célébrer ses exploits guerriers (v. 682-1228). Elle montrait aussi comment l'auteur s'employait déjà à relier son texte aux poèmes suivants, ceux de la première Croisade. La projection dans le passé des origines, une génération avant Elias, d'une expédition défensive contre des Sarrasins concourait sans doute à prouver que le lignage, dès sa fondation, avait pour vocation d'anéantir les païens. Or, l'auteur de la Chanson, tout en réhabilitant le roi, ne reprend pas cette voie explorée par Elioxe. S'il justifie lui aussi le départ du roi par les contraintes de la guerre, il préfère signaler qu'il abrège cette séquence de combats, comme si elle ne l'intéressait pas :

Ly boins roys Orians ne s'y vot ariester ;

Avoecques ses barons se vot acheminer ;

En la guerre s'en va pour son païs sauver,

Encontre .I. roy payen, que Dieux puist craventer! 
De la guerre qu'il fist ne vous say deviser.

$\mathrm{Ne}$ ja viers vous n'en voel la cançon arrierer :

Ains revenray au fait pour l'istor abriéver,

Ensi que la cronicque le nous fait raconter. (v. 252-259)

croisades se confirme un peu plus loin. Au terme de son adaptation de Beatrix, l'auteur supprime en effet un autre épisode guerrier, les combats contre les Sarrasins dans lesquels Elias s'illustre au cours de sa navigation vers Nimègue (Beatrix, v. 2688-3102). La célébration de ces hauts-faits de chevalier chrétien, une invention de l'auteur de Beatrix, s'inspire pourtant elle aussi de très près des chansons de croisade, notamment lorsque des troupes divines viennent secourir l'élu de Dieu, avec saint Georges à leur tête :

Les galies aprocent pour Elias aidierQue Dex li envoia qui tout a a jugier.Sains Jores les conduist devant el cief premier,Entre angeles et arcangeles i a plus d'un millier,Tout sunt blanc revestu con prestre de moustier,Vers Elias s'en vienent tant con porent nagier.Li cisnes fait grant joie qant les vit aprocier.Lors s'entrevienent tout sans traire et sans lancier.La mesnie saint Jore vont iceus essaierEt li cisnes lor vait lor galies percier,A son bec les descoupe por le mius empirierQu'il ne set autrement parler ne losengier.

Les galies aprocent pour souscorre l'enfantQue Dex lor envoia par son digne coumant.Durement les asalent et cascuns se desfent.Une galie afondrent de la jent Tervagant,En l'aighe sunt ceü et noié plus de .C. ;Toute est enplie d'aighe, au fons va tournant.Li autre se desfendent mout angousousemant,La mesnie saint Jorge les grieve duremant.Este vous .I. orage qui la mer va torblantEt li galiot tornent par mi la mer fuiant.Il n'ont gaires alé quant tounoiles les prentQui se fiert es galies, tous les va craventant ;Or ont assés alé li cuvert mescreantEt les blances galies s'en retornent atantQue Dex i envoia par son digne coumant. (Beatrix, v. 2708-2735)

41 Le lecteur peut ainsi s'étonner de l'absence dans la Chanson de ces épisodes triomphants de croisade au sens large du terme, qui, tout en donnant une intensité épique, pouvaient expliquer la prédestination du lignage à reprendre Jérusalem durant la première Croisade. Mais si l'auteur du XIV ${ }^{\mathrm{e}}$ siècle refuse de prêter des exploits contre les Sarrasins aussi bien à Elias qu'au père d'Elias, c'est peut-être pour que Godefroy de Bouillon apparaisse ensuite comme le premier héros de la croisade, pour lui réserver cette primauté. Difficile aussi pour lui d'imaginer une prise mythique de Jérusalem deux générations avant la première Croisade... Elias est donc célébré comme un héros pieux, un chevalier chrétien exemplaire, mais non comme une préfiguration des Croisés. Plus loin, dans le Chevalier au Cygne, ses combats contre les Saxons révèlent à nouveau son élection divine, mais ils ne prennent jamais non plus la signification d'une croisade : Dieu prédestine Elias à la victoire pour que la justice triomphe et surtout pour qu'ensuite il régénère un lignage tombé en quenouille et lègue à ses descendants une relation privilégiée avec Dieu, qui leur assure le succès en Terre sainte. L'accent mis sur la piété du héros se renforce d'ailleurs dans la Fin d'Elias et son adaptation dans 
la Chanson, lorsqu'Elias, de retour dans son univers après la transgression de l'interdit par la duchesse, se retire dans un monastère. La Fin d'Elias lève alors le tabou qui pesait jusqu'alors sur la connaissance de ses origines, elle révèle le devenir du héros mélusinien après son départ. Or, cet Autre monde dans lequel les créatures féeriques sont habituellement contraintes de retourner, c'est ici le royaume paternel du roi Oriant, qui appartenait jusqu'alors au monde des hommes : les frontières entre la féerie et les réalités humaines se brouillent à nouveau. Les deux mondes n'en restent pas moins irrémédiablement séparés, non parce qu'Elias jouirait d'un statut ontologique autre ou qu'il serait soumis à une malédiction comparable à celle de la fée Mélusine, mais parce qu'il se soumet à la volonté de Dieu et consacre la fin de son existence à son service exclusif, dans la solitude. Comment mieux dire la vocation religieuse du lignage ? L'ancêtre de Godefroy, le héros fondateur de la famille, meurt tel un saint. Son étrangeté, loin de la féerie, émane de sa sanctification, sa piété s'exprime selon un idéal religieux très exigeant mais différent de celui de la croisade.

La suppression des combats du Chevalier au Cygne contre des Sarrasins et la réhabilitation de son père s'accompagnent enfin d'une modification très profonde du portrait de Matabrune. La grand-mère d'Elias perd la fureur démoniaque et la sauvagerie incontrôlable qui la caractérisent dans Beatrix. La Chanson tend à la confiner dans son seul rôle narratif d'opposant, sans jamais lui permettre de devenir l'héroïne principale, celle qui détermine le cours des événements dans Beatrix, monopolise l'attention, subjugue les consciences. Comme nous l'avons rappelé plus haut, la critique a jusqu'à présent valorisé les facteurs de cohérence qui existent bel et bien entre Beatrix, le Chevalier au Cygne et les poèmes suivants du cycle, au point d'estimer que Beatrix serait à tous égards une meilleure continuation "par l'avant " qu'Elioxe. Peutêtre convient-il aussi de montrer que la version de Beatrix se construit néanmoins sur une tension a priori excessive, car en partie inadéquate à la signification d'ensemble du cycle. En créant un univers manichéen, où les forces du bien et celles du mal s'affrontent violemment, son auteur montre certes sa fidélité à la tradition épique médiévale et annonce les oppositions sans nuance qui structurent les poèmes de la première Croisade. Mais dans Beatrix, ce qui est différent, c'est que le mal s'incarne dans un personnage très proche du Chevalier au Cygne par les liens du sang, ces liens qui justement fondent le lignage : sa grand-mère Matabrune. Renouant avec la violence misogyne du Dolopathos et noircissant à l'extrême son portrait, il la transforme en un véritable suppôt du diable. Tandis qu'Elioxe ignorait ce surnaturel démoniaque, il n'a de cesse de confirmer l'appartenance à l'univers diabolique de « la vieille Matabrune ki en Jhesu ne croit » (v. 101), un « maufé » (v. 175), un « avresier » (v. 224) :

Sa mere ert .I. diables pour le mont encanter. (v. 43)La vielle se pourpense c'un grant mordre feroit,Diables le soumont cui oevre ele faisoit. (v. 112-113)

Sa violence irrépressible, la terreur qu'elle inspire et la soumission qu'elle impose révèlent sa nature de démon :

Quant li preudom l'entent tout a le sanc müé,Ne l'ose contredire tant doute le maufé,Se li a otroié toute sa volenté. (v. 174-176)Li sire a tel paour quant l'avresier ententQue il ne set que dire tant a le cuer dolent. (v. 224-225)

Plus loin, alors que les serfs qui maltraitent Beatrix «par le plaisir de Deu la veüe ont perdue» (v. 289), les diables empêchent Matabrune de subir le même châtiment: «mais cele ki toute a la folie meüe/n'en a nul mal eü, diable l'ont tenue » (v. 291-292). L'auteur multiplie tant ses actes de violence qu'elle devient l'héroïne principale de sa version. Elle aveugle le serviteur qui lui a désobéi, elle prend les armes contre son 
propre petit-fils, puis sur le bûcher, dans une confession sans repentir, elle revendique le mal pour le mal et recommande son âme aux diables :

«Or oiés», fait la vielle, "cevalier et baron.Ja orés grant mervelle en ma confiessïon.A .I. mot vous puis dire! Ainc ne fis se mal non !Jou n'amai onques Dieu ne son saintisme non,Onques riens tant n'amai con mordre et traïson.Se peuise encor vivre, foi que doi saint Simon,Jou copase Eliase le cief sor le mentonEt mon fil ocesise ancois l'Asentïon.Puis mesise la tiere a fu et a carbon,Si fesise ses houmes traire male saisonEt raensise tous et mesise en prison ». (v. 2493-2403)《 As diables d'infier soit m'arme coumandeeEt tout cil ki ci sunt dedens ceste contree! »Lors s'estendi la vielle, toute est arse et brulee ;L'arme enportent diable, a aus estoit vöee. (v. 2526-2529)

Bien entendu la logique narrative du conte des enfants-cygnes lui assignait nécessairement le rôle d'opposant, mais l'auteur n'était pas tenu de renforcer la perversité qui la caractérise déjà dans le Dolopathos. Preuve en est d'ailleurs la version parallèle d'Elioxe, qui montre au contraire un «adoucissement» du personnage : avec les transformations apportées au schéma narratif, Matabrune ne peut imposer un martyr à la jeune reine, puisque cette dernière est destinée à mourir en couches selon une volonté supérieure qui est, semble-t-il, celle de Dieu. L'invention de la mort prématurée de la jeune mère permet donc de donner moins d'importance à la bellemère : après la révélation de la vérité, elle est rapidement terrassée et condamnée au bûcher.

Dans Beatrix, la diabolisation de Matabrune nourrit en revanche très efficacement la dramatisation du récit et, par contraste, contribue à révéler avec éclat l'élection divine du Chevalier au Cygne. L'effacement du surnaturel féerique d'inspiration païenne se conjugue donc avec un épanouissement du surnaturel chrétien, déjà présent dans Elioxe, mais aussi du surnaturel démoniaque, ce qui est plus surprenant. Vu l'étroitesse du lien familial qui unit Matabrune à Elias, il pouvait en effet apparaître peu judicieux, ou tout au moins problématique, dans une perspective de célébration du lignage, d'accorder trop d'ampleur à sa propension au mal. Autrement dit, à l'ancêtre féerique maternel, héroïne principale d'Elioxe, est substituée une grand-mère démone a priori plus difficile à concilier avec la christianisation du Chevalier au Cygne, bien que le conflit qui l'oppose à son petit-fils ait justement pour conséquence de prouver les liens qui unissent le jeune homme à Dieu. Force est néanmoins de constater que l'auteur de Beatrix ne soulève jamais la question de cette ascendance très inquiétante et des soupçons que ces liens du sang pourraient faire peser sur le Chevalier au Cygne. Faut-il rappeler à quel point les légendes sur des fils ou filles de diables imprègnent les mentalités médiévales? Pensons aux efforts que Jean d'Arras déploie pour nous convaincre que la fée Mélusine, malgré sa transformation en serpente, n'est pas une démone ou bien, dans les Romans d'Alexandre, aux doutes que les accusations d'adultère d'Olympias avec un enchanteur nourrissent sur la possible appartenance à l'univers diabolique d'Alexandre le Grand. Le point de vue de l'auteur de Beatrix est autre, mais son récit, en dépit d'un manichéisme insistant, est traversé par les ombres que jettent plusieurs ambiguïtés, à commencer par les conséquences de ce statut ontologique très inquiétant de la « vieille».

Tout se passe comme si l'auteur de la Chanson du XIV e siècle avait bien pris la mesure de cette promotion du surnaturel démoniaque dans Beatrix, puisqu'il supprime, non pas tous les actes de violence, mais toutes les marques explicites de l'appartenance de Matabrune à l'univers du diable. Ainsi travaille-t-il à un rabaissement du personnage, 
qui perd son origine surnaturelle et les pouvoirs très inquiétants qu'elle lui donnait, si bien que, lors de l'ultime affrontement, elle est tout de suite vaincue par son petit-fils, sans les combats acharnés que relatait Beatrix, sans la scène haute en couleurs de la « vieille au combat » contre son propre lignage. En voici pour autre preuve l'évocation, très abrégée et très sobre, de ses dernières paroles, qui expriment désormais le pardon et presque le repentir, puis de sa mort, qui ne provoque l'intervention d'aucun démon:

A Helyas a dit: "Biaus fieux, a moy venés.J'ay bien desirvit mort, c'est fine verités ;Vostre mere ay trahy, dont che fu grans pités;VII enfans li cangay qu'elle avoit portés,A VII kaines d'argent, et se li mis de lésVII kiens, d'une lisse tous nouviaus kaielés.Je vous pardoins ma mort, mais que me delivrés. »Matabrune fu arse a grant destructionPar devant Maubruiant, le nobile maison. (v. 2238-2245)

Deux autres points troubles disparaissent également: les liens de Matabrune avec les Sarrasins et la connaissance qu'elle semble détenir de la vertu des chaînes merveilleuses. Nous avons rappelé plus haut que, dans sa pratique de la continuation «par l'avant» et son souci d'annoncer les épopées de la croisade, l'auteur de Beatrix attribue à son héros des combats contre des Sarrasins inconnus des versions antérieures. Une raison supplémentaire explique peut-être leur occultation dans la Chanson: une note dissonante retentit en effet dans ce bel épisode d'inspiration épique qui semble à première vue en adéquation idéale avec les poèmes sur la première Croisade. Elle résonne même si fort qu'elle met à mal l'harmonie apparente de la fin de Beatrix. Les dernières troupes sarrasines qu'affronte Elias, celles qui combattent au nom de Tervagant et de Mahomet, ont pour chef Agoulant. Or ce dernier est inventé comme le propre frère de Matabrune, soit le grand-oncle d'Elias. Les Sarrasins ne constituent donc pas une figure de l'Autre absolu, ils appartiennent au lignage lui-même, à ses origines les plus lointaines, celles de Matabrune. Emporté par sa volonté de condamner radicalement Matabrune et d'idéaliser Elias comme chevalier de Dieu, l'auteur de Beatrix imagine une scène nouvelle qui le conduit jusqu'au paradoxe: l'épisode se construit bien à partir de scénarios et de motifs hérités des chansons de geste, mais il associe l'affrontement à un lien familial difficilement compatible avec l'opposition manichéenne que l'auteur cherche à tracer et avec l'univers de valeurs épiques dont il s'inspire. À la fin du Moyen Âge, l'écrivain de la Chanson n'est d'ailleurs pas le seul à en ressentir les difficultés d'interprétation. Le prosateur du XVe siècle, Berthault de Villebresme, choisit aussi de le supprimer, comme si, à ses yeux également, il était impossible que les origines du lignage de Bouillon réunissent pour les opposer chrétiens et musulmans.

Dans Beatrix, les pouvoirs démoniaques de Matabrune, au-delà de ses actes maléfiques, de ses liens familiaux avec des Sarrasins et des déclarations du narrateur, sont également suggérés par la connaissance qu'elle semble mystérieusement acquérir de la capacité de métamorphose des enfants. Alors que la vertu des chaînes n'a pas encore été révélée, comment peut-elle la deviner, si ce n'est grâce à un savoir surnaturel, celui d'une démone? Il n'est certes jamais déclaré clairement qu'elle chercherait en toute connaissance de cause à provoquer la transformation des enfants en cygnes, mais rien n'explique pourquoi, toute à son désir de se débarrasser à jamais des enfants, elle ordonne à son serviteur de leur dérober les chaînes plutôt que de les tuer. Le lecteur subodore alors qu'elle décide consciemment d'éliminer sa descendance en la contraignant à perdre sa forme humaine, ce qui lui permet en outre d'assouvir sa convoitise en s'enrichissant de ces joyaux extraordinaires. Dans Beatrix, comme déjà dans Elioxe, la métamorphose n'est plus interprétée comme un héritage de la figure 
maternelle étrangère, mais comme un don de Dieu, ce qui ne pose en apparence aucun problème théologique, d'autant que par ailleurs les auteurs rappellent très souvent que Dieu a créé l'homme à son image. En même temps, à partir d'Elioxe et dans toutes les versions suivantes, les enfants ignorent l'existence de ce don jusqu'à leur propre transformation. Ils ne peuvent donc pas et ne pourront jamais passer volontairement d'une apparence à l'autre et tirer un éventuel profit de cette double nature, comme le chevalier-oiseau Yonec en avait la capacité dans le lai de Marie de France par exemple. Cette double nature ne leur offre pas une puissance supérieure. Leur nature animale sommeille en eux telle une potentialité, si enfouie que la connaissance leur en échappe et surtout qu'elle n'aurait jamais dû se révéler s'ils n'avaient pas été confrontés à Matabrune. Bien que rendue possible par Dieu, la métamorphose en animal n'est pas réalisée par Dieu. Seul un personnage inhumain, voire diabolique, peut la provoquer et, dans une perspective chrétienne, l'auteur de Beatrix suggère ainsi que le démon n'a pas le pouvoir de transformer la création divine, mais doit se contenter d'exploiter ce qui émane de Dieu. Lorsque la Fin d'Elias relate plus loin la cérémonie chrétienne qui permet la muance inverse, de cygne en homme, au bénéfice du malheureux frère dont Matabrune a fait fondre la chaîne, c'est en revanche Dieu qui efface la forme animale et redonne au cygne sa dignité humaine.

Impossible donc de savoir avec certitude si la Matabrune de Beatrix connaissait la vertu des chaînes quand elle demande au serviteur de les couper, mais force est de constater que, quand elle avait vraiment voulu provoquer la mort des enfants, à leur naissance, alors même qu'elle avait déjà constaté la richesse de ces chaînes, elle avait exigé leur mort. Il serait donc tentant de penser qu'elle devine le pouvoir de ces bijoux et décide de l'activer pour punir les enfants d'être restés en vie : ce serait alors une surenchère dans la cruauté, puisque la métamorphose animale provoque des tourments plus douloureux que la mort par leur durée et qu'elle prend bien soin de donner les chaînes à fondre, comme s'il s'agissait de supprimer tout espoir de retour à la condition humaine. Matabrune ne cesse d'ailleurs de rabaisser ses victimes à la condition animale dans Beatrix. Après avoir accusé la jeune reine de relations sexuelles avec des chiens et de l'avoir traitée comme une chienne, tout se passe aussi comme si elle la frappait de sortilèges démoniaques : la privation de la parole rappelle la geis des récits féeriques. La "vieille» est ensuite directement responsable de la métamorphose effective des enfants en cygnes. Seul Elias y échappe "par hasard ", mais les contraintes de la vie dans la forêt l'entraînent à régresser dans son aspect physique à l'état animal. Enfant sauvage, il ressemble à un loup et, tel Perceval, ignore tous les usages sociaux. Néanmoins, rien n'est dit sur sa chaîne dans Beatrix : à partir du moment où il apprend le sort de ses frères et de sa sœur, l'existence de cet attribut surnaturel est occultée, comme si la connaissance faisait disparaître sa double nature potentielle et le libérait de cette faiblesse.

51 Dans sa relecture de Beatrix, l'auteur de la Chanson du XIV e siècle semble avoir poursuivi sa réflexion sur les conséquences de l'attribution d'une telle ancêtre diabolique à Godefroy de Bouillon, puisqu'il amoindrit encore son pouvoir en supprimant ce qui pouvait laisser penser qu'elle disposait d'un savoir surhumain, un savoir qui lui permettait de connaître les secrets de Dieu et de les exploiter à son profit. Ainsi modifie-t-il quelques éléments de sa source qui peuvent d'abord apparaître comme des détails mais sont en réalité lourds de signification, et ajoute-t-il des indices qui suggèrent que Matabrune ignore la vertu des chaînes. Lorsqu'elle les découvre à la 
naissance des enfants, elle les interprète clairement en mauvaise part, comme un signe de vilenie qui annoncerait les crimes et délits que les enfants commettraient:

C'est un signes de Dieu qui cha jus est moustrés, Que s'il vivent lonc temps, bien croire le porés, Qu'il seront mourdreour et fel laron prouvés :Si qu'il vaut assés mieulx que on les ait gitésDedens une riviere, noyés et effondrés. (v. 415-419)

Puis, l'auteur transforme le discours qu'elle adresse à son serviteur, une fois qu'elle apprend que les enfants ont survécu: elle n'a plus jamais l'intention de leur dérober leurs chaînes, dont elle n'a d'ailleurs pas été frappée par la richesse, mais exige une seconde fois leur mort (v. 781-782) ${ }^{23}$. L'adaptateur invente alors tout un faisceau de circonstances nouvelles qui apportent une explication vraisemblable au vol des bijoux. Le serviteur de la méchante reine, tandis qu'il se rend auprès des enfants pour les assassiner, croise une procession qui conduit au bûcher une mère accusée d'infanticide. La peur de l'infamie et de la damnation s'éveille en lui, à tel point qu'il prend l'initiative du vol des chaînes pour épargner les enfants, et l'on remarque avec quel soin l'auteur imagine un enchaînement cohérent d'événements précis :

Enssy que Savaris par ung hamiel passaVit moult de gent issir pour ung tant c'on alaVir une femme ardoir c'uns bouriaus amena.Le fait qu'elle avoit fait Savaris demanda,Et uns varlet li dist: «Ne vous mentiray ja,Mourdry a son enfant que li siens corps porta. "Quant Savaris l'oy, tous ly sans li mua :Des enfans ly souvient, pourquoy il venoit la,Dist a ses compaignons : « Ung biel miroir chi aMatabrune no dame, qui chy tramis noz aPour mourdrir les enfants; trop mal nous consella.Honnis soit-il de Dieu, qui ja mal leur fera!Regardés ceste femme qui tantos ardera,Mourdry a son enfant, de li me souvenra, « Cha! dist ly braqueniers, savés que nous ferons ?Tos et isnielement as VII enfans irons,Les kaines de leurs cols tantos leur r'osterons,Et puis a Matabrune nous les raporterons,Et che seront enseignes que destruis les arons. » (v. 802-820)

Dans la stupéfaction la plus totale, le serviteur assiste ensuite à la métamorphose et à l'envol des enfants, qu'il ne révèle jamais à sa maîtresse, pour cacher sa trahison. Dans la réécriture $\mathrm{du} \mathrm{XIV}^{\mathrm{e}}$ siècle, la scène de métamorphose n'est donc plus reliée à l'intervention d'un être démoniaque, comme s'il s'agissait, non seulement de rabaisser Matabrune, mais aussi de garder toute sa pureté à la «naissance » sublime des cygnes : "trestout li vi enfant sont en chine mué,/par le voloir de Dieu, le roy de magesté » (v. 846-847). La métamorphose est désormais tout entière associée à la puissance divine. Que la « muance » entraîne le passage de l'homme à l'animal ou de l'animal à l'homme, elle n'appartient qu'à Dieu, elle n'est plus "souillée » par le savoir et le pouvoir que pourrait détenir une créature du diable. L'auteur du XIV siècle innove aussi en ajoutant une scène fugace qui donne une profondeur humaine nouvelle à Elias. Lorsqu'il apprend le sort de ses frères et de sa sœur, le premier souhait du futur Chevalier au Cygne est de les rejoindre :

Il a dit a l'iermite : «Sire, je vous creans; Puisque me frere sont ensi chisne noant,Je voel r'oster me kaine qui au col m'est pendant:Chisnes voel devenir, trop le sui desirans ; Car ce sont biel oisiel, gracieus et plaisans.S'iray avec mes freres noer, il en est temps. "Sa kaine euist r'osté Helias, li vaillans,Se ne fust li preudons qui li fu deffendans :Et dist : « Layés ester et n'i soiés penssans.Ains tel mesquief n'avint puis que fais fu Adans; Car une gieste doit venir de ces enfansDont la foi Jhesucris en sera mieulx creans.«Biaus fieux, dist li preudons, pour Dieux et pour son non,Enteng ma volenté et s'acorde men bon.Ly chisne revenront en luer propre fachon,Li chisne gracieux de ten estracion,Et seront trestout roy et prinche de renon,Et istera de vous Godefroy de Buillon,Qui de Jherusalem maintera le roïon. (v. 1151-1169) 
Ainsi aurait-il provoqué sa propre métamorphose en arrachant sa chaîne si l'ermite ne l'en avait empêché pour lui apprendre sa mission. Dieu lui interdit donc de changer de nature et de passer librement d'une apparence à une autre comme les créatures de l'Autre Monde en ont le pouvoir dans les récits féeriques.

L'auteur de la Chanson du XIV ${ }^{\mathrm{e}}$ siècle, qui donne à la légende de la naissance du Chevalier au Cygne son ultime postérité littéraire en laisses épiques, ne cesse ainsi de modifier ses deux sources d'inspiration, Elioxe puis Beatrix. En comparaison, la mise en prose $d u X V^{e}$ siècle paraît plus conservatrice, en dépit du choix de cette forme d'écriture "à la mode ", creuset de tous les univers littéraires à la fin du Moyen Âge. L'auteur explique d'ailleurs qu'il ne souhaite que «mectre et translater icellui livre et memoriable histoire de ladicte ancienne rime et obscure lengaige en prose et lengaige françois cler et entendible» (p. 2). Le changement de forme littéraire est donc légitimé par le vieillissement du français du XIII siècle et une volonté de traduire dans une langue plus moderne. Le prologue se clôt ensuite sur une affirmation de fidélité au contenu de son unique source, Beatrix : «l'extrairay et translateray sans y adjouster ne mectre sinom aucuns petiz notables et moriaulx advertissemens ou il me semblera estre expedient, duisant et convenable»(p.3). Il annonce ainsi une exploitation moralisante de l'histoire héritée, qui se manifeste plus loin par des interventions incessantes du narrateur, dont les jugements, les indignations, les apitoiements amplifient le récit. Voici un premier exemple qui caractérise bien sa technique de réécriture. Alors que Beatrix ne consacre que quelques vers à l'évocation de la pitié du serviteur qui, chargé par Matabrune de tuer les nourrissons, décide de les épargner (v. 308-318), le prosateur amplifie la scène, en ajoutant ce long commentaire :

Icy peult on congnoistre que souvent on trouve plus de pitié et d'amour en estranges gens que on ne fait en ses propres parens, car ses sept petiz enffans, naguerez nez et nouvellement venuz au monde, furent par Mathebrune, leur ayeule paternelle, baillez a Macaire, homme serf, pour estre noiez en ung vivier et puis enterrez, mais ils trouverent en Macaire la pitié et compassion qu'ilz ne trouverent pas en Mathebrune leur ayeule, car il les envelopa et les laissa sur le rivaige sans leur faire aucun mal ne desplaisir. Hellas, ces jeunes enffans essaierent bien toust le tournoiement et la durté de Fortune, laquelle n'atendit pas qu'ilz sceussent aller et parler pour leur faire savoir quel jeu elle scet jouer. Mais, incontinent qu'ilz furent nez, elle leur fut sy felle et estrange qu'elle les fist mectre hors de la chambre de la royne leur mere et mectre en leur lieu sept chiens, et souffrir plusieurs aultres miseres et pouvretez-comme vous orrez cy apres. Mais, bien souvent, ceulx qui sont si infortunez et mal traictiez en jeunesce sont a la fin plus eslevez et viennent a plus grande perfection que ceulx qui mignotement et souefvement sont par grande curançon et diligence nourriz et chiers tenuz, ainsi qu'il appert en plusieurs anciennes histoires, meismes en l'histoire presente, et le voit on chacun jour par experience. (pp. 11-12).

Plutôt que de réinterpréter le récit dont il s'inspire, il préfère ainsi très souvent adopter le point de vue d'un moraliste, en tirant la leçon, morale ou politique, des événements relatés ou en dramatisant le récit par des annonces qui rappellent les prolepses des chansons de geste.

Sa mise en prose commence par suivre scrupuleusement Beatrix, comme si le changement de facture, sans se limiter à un simple dérimage, autorisait néanmoins davantage la reproduction du même. C'est donc la parole calomnieuse de Beatrix qui est à l'origine du récit, avant que l'évocation de l'accouchement ne soit brièvement exploitée comme un exemple qui prouve le caractère naturel et conforme à la volonté 
divine des naissances multiples (p. 5). Impossible de savoir si l'auteur connaissait aussi la Chanson du Chevalier au Cygne et de Godefroy de Bouillon, mais il introduit un élément qui semble spécifique à cette adaptation du XIV e siècle: Beatrix n'a pas pu voir ses propres enfants (p.6) et les accusations que lui lance Matabrune provoquent son évanouissement. La parole lui est retirée. Elle ne la retrouve que pour prononcer une prière à voix basse (p. 8), mais la honte l'empêche d'essayer de se défendre (« excuser ne se povoit comme celle qui ne savoit la verité de son enffantement»(p.9)). Aucun signe de féerie n'apparaît alors. Le seul que conservait Beatrix, tout en l'associant au surnaturel divin - la mention des sept fées qui auraient programmé le destin des enfants et leur auraient donné les chaînes - est éliminé, au profit d'une christianisation plus complète :

La dame se delivre a duel et a destroitL'un enfant apres l'autre, si con Dex le voloit.Al nestre des enfans .VII. fees i avoitQui les enfans faerent que cascun avenroit,Ensi que li .I. enfes apres l'autre naiscoit,Au col une caïne de boin argent avoit. (v. 102-107)

Puis apres ne tarda gueres que la royne enfantta et eut six beaulx filz et une belle fille, lesquieulx, ainsy qu'il pleut a Dieu, qui en ses euvres fait souvant choses merveilleuses et incomprehensibles a humain entendement, avoient chacun une chesne d'argent au col. (p. 4)

Des modifications affectent ensuite le portrait du père d'Elias et de sa grand-mère Matabrune, quoiqu'elles renouvellent moins profondément l'image des origines du Chevalier au Cygne que dans la Chanson du XIV ${ }^{e}$ siècle. L'auteur humanise le roi Oriant en lui prêtant de déchirantes manifestations de douleur (p.9), puis il lui donne longuement la parole dans un émouvant discours qu'il adresse à sa femme (pp. 9-10). Mais la réhabilitation du personnage constatée dans la Chanson n'a pas lieu, car, conformément à Beatrix, le roi reste sous la complète domination de sa mère, qu'il ne peut empêcher de faire violence à Beatrix. Avant de la condamner au bûcher, il tente certes d'organiser un procès. Cependant, la scène de jugement se déroule bien autrement que dans la Chanson. Aucun évêque n'intervient avec autorité pour prendre la défense de la jeune femme. Les barons, dénués de tout sens de la justice, flattent le roi en approuvant à l'avance sa décision et lui, incapable de se prendre position, telle une marionnette qui se laisse manipuler, s'en remet entièrement à sa mère :

"Mais vous ", dist il a Mathebrune, « qui sçavez la verité du cas, se elle l'a commis ou non, jugez la ». O ! bon Dieu, quelle parolle de roy! Commectre le jugement de la royne, sa femme, a Mathebrune, son ennemye mortelle, qui seulle l'avoit accusee et qui par toutes les cautelles, subtillitez et malices qu'elle povoit controuver et dire, prouchassoit sa mort et exterminacion. Certes ce fut simplement advisé au roy Orient et mal digeré ses parolles par lesquelles il monstra qu'il estoit pou sçavent es droitz et loitz civilles, qui privent et estrangent toutes femmes d'office, de judicature et de tous aultres offices civilz et publicques, et aussi tous hommes et femmes es causes ou ilz font partie comme estoit Mathebrune contre la bonne royne. Mais pour aucunement excuser le roy, il est a croire qu'il estoit sy troublé pour la singulliere et grand amour qu'il avoit a la royne, et pour l'orribleté du cas que sa mere luy imposoit et aussi pour la continuelle importunité d'elle, qui incessanment le sollicitoit de faire mourir la royne, qu'il ne savoit qu'il diroit, ainsi qu'il advient souvent a gens qui sont fort esmeuz, courroussez et agravez de dueil (p. 19).

59 Comme Beatrix ne relatait aucune scène de jugement, le prosateur ne se souvient-il pas ici de l'adaptation du XIV ${ }^{e}$ siècle? Si tel était le cas, sa version signifierait qu'il a consciemment adopté un point de vue inverse. La mise en prose dénonce en effet l'incapacité d'Oriant à déchiffrer la situation, à analyser le comportement de ses 
proches et à remplir ses devoirs de roi. L'auteur condamne sa démission et même s'en indigne, tout en essayant d'adoucir son verdict par quelques circonstances atténuantes. Au-delà du recours à l'explication psychologique et de la condamnation de Matabrune, la lâcheté des barons est ensuite stigmatisée, car ils entérinent l'injustice en toute connaissance de cause :

Quant Mathebrune eut prononcee sa dempnale sentence, les barons qui la estoient presens s'accorderent a la tenir, puis que le roy lui en avoit baillé la charge combien qu'ilz congneussent le jugement estre inique et desraisonnable - et n'oserent, ou ne voullurent, contester a Mathebrune, ainsy que font souvent plusieurs, lesquielx, ja soit ce qu'ilz voient et congnoissent le tort qu'on fait souventesfoiz a pouvres gens innocens et miserables personnes, le tollerent et dissimulent, sans mot sonner, pour peur d'encourir le malle grace de de leurs maistres et seigneurs. Et a cause de ce sont plusieurs maulx et adviennent de grans inconveniens, lesquelx n'aviendroient pas se en temps et lieu verité estoit dicte et remonstree. (p. 20)

60 La séquence délivre donc un message politique, elle illustre les conséquences d'un mauvais exercice du pouvoir royal et de la faiblesse coupable des courtisans.

Đlus loin, le récit de la résistance de Matabrune et de son recours à l'argent pour stimuler le zèle de ses soldats est exploité comme un exemple qui prouve les effets tragiques d'un usage pervers de la largesse, ce qui permet d'introduire un rappel à la Chanson de Roland et de dresser un parallèle entre Matabrune et le roi Marsile :

Ainsy appert qu'il n'est mauvaistié ne trahyson qu'on ne face par avarice et pour argent avoir. Par ceste mauldicte avarice et pour les grans dons que le roy Marseille donna a Gannes, il trahit les douze pers de France qui piteusement, a cause de celle trahison, mourent a la bataille de Roncevaulx ; et tant d'aultres et infiniz maulx en sont advenuz, que les anciennes histoires en sont toutes plaines, et chacun jour en adviennent, ainsi que on voit par experience, et est une lamentable, piteuse et dampnable maniere de vivre, et d'avoir et acquerir des biens par telles trahisons et exquises mauvaistiés, dont la fain est toujours dolloureuse et miserable, mesmement pour ceulx qui les font, car on dit communement que trahison retourne tousjours a son maistre. (p. 43)

62 À la clôture de sa mise en prose de Beatrix, avant l'évocation du départ du Chevalier au Cygne pour Nimègue, l'auteur revendique une nouvelle fois la subordination du récit à la morale :

En cest maniere fina miserablement ses piteux et lamentables jours la mauvaise et maleureuse Mathebrune, a sa grant honte et confusion et deshonneur, selon la desserte de ses vilains, ors et detestables fais : qui est ung bel, moral et fructueulx exemple a toutes dames, damoiselles et aultres, tant hommes que femmes, de quelque estat ou condicion qu'ilz soient, pour eulx bien vertueusement et honnestement gouverner, sans faire aucune injure, trahison, villenie ou deshonneur ; ne impouser aucun crime, blame ou reprouche a aultruy, affin que par leur bonne et honneste conduite et vertueuse maniere de vivre, ilz puissent acquerir l'amour de Dieu et du monde et, finablement, la gloire de paradis; et eviter, esloinger et fouir uune telle si confusible, honteuse et vituperable mort que souffrit, a bon droit et raison, la malureuse viesle Mathebrune, combien qu'elle feust royne et grant terrienne. (p. 49)

63 Auparavant, les souffrances endurées par la mère ont été réinterprétées comme des épreuves envoyées par Dieu et sa délivrance finale comme une marque du soutien que Dieu finit toujours par accorder à ses fidèles serviteurs :

Lesquelles choses elle avoit humblement et pacienment endurees, par quoy Dieu, qui souventesfoiz envoye a plusieurs bonnes creatures diverses mondaines 
persecucions et adversitez pour esprouver leur bonne constance, les miseres et adversitez que ceste pouvre royne avoit eues et pacienment endurees, ne l'oublya pas a son grant besoing, mais le fist par Helyas, son filz jeune, foible et tendre, miraculeusement delivrer. Ainsi ne se doit aucun desesperer ne deffier de la grace et misericorde de Dieu quant aucune adversité lui survient, soit a tort soit a droit, mais pacienment et doulcement l'endurer par l'amour de lui qui tant souffrit et endura pour nous. Car a la fin la verité se congnoist, et Dieu aide tousjours a ceulx qui ont bonne esperance et pacience, ainsi qu'il fist a ceste bonne et honneste royne (pp. 32-33).

64 Cet élargissement constant du propos, avec le passage du singulier à l'universel, entraîne ici Berthault de Villebresme à recourir à une explication convenue qui fausse la signification de l'histoire. Difficile en effet d'assimiler les persécutions infligées par Matabrune à une mise à l'épreuve divine, car rien n'indique que Matabrune puisse être à son insu un instrument de Dieu.

À la lecture de la mise en prose, on constate néanmoins un infléchissement du portrait de Matabrune qui ne s'explique par seulement par les exigences d'une interprétation moralisante, moyen commode d'amplifier le récit en vers. Si Matabrune est condamnée pour son inhumanité et sa perversité, les signes de son appartenance au monde du diable et de son statut ontologique de démon disparaissent. Elle n'en demeure pas moins d'une cruauté redoutable. L'image dominante que le prosateur trace d'elle, c'est celle d'« une femme desnaturee et differante a la commune condicion de tous ayeuls et ayeules », car coupable de trahir son propre lignage. Elle s'en prend ainsi à Elias dès son arrivée à la cour, en lui griffant le visage jusqu'au sang (p. 27), puis, retranchée dans son château, finit par l'affronter ellemême, comme dans Beatrix :

Puis monta Helyaas ou chastel, ou il la trouva; laquelle, voiant ces gens occis, son chastel conquesté, ses ennemys dedans, et que eschapper ne povoit aucunement qu'elle ne feust prinse, estoit sy despite et desesperee qu'elle sembloit ung ennemy d'enfer. Et a haulx et espoventables criz appelloit tous les dyables ses maistres en son aide. Quant Helyas la vit, il appella ses gens, et leur dist: «Prenez moy ceste viesle enragee. » Elle l'ouït, si vint vers lui, les sourciz levés, roullant les yeulx, ternissant son vis comme ung tigre ou lion familleux qui envahit sa proye. Et a ses deulx poings, qu'elle avoit grans, larges et estanduz, le saisit rudement et le cuida geter a terre. Mais Helias, qui bien se savoit deffendre, la print par les cheveulx, qu'elle avoit longs, noirs, et laiz et hideux, et la demena si lordement qu'i la gecta a terre, si roidement que le sang luy sailloit par la bouche et par le néz. (p. 46)

Berthault n'a donc pas cherché à gommer la violence de l'affrontement, mais Matabrune est seulement comparée à un diable. La comparaison remplace la véritable assimilation du personnage à un démon à laquelle on assistait dans Beatrix. Plus encore, la Matabrune de la mise en prose a beau appeler les démons, ils n'accourent pas à son aide comme Beatrix le montrait parfois, pas plus qu'ils ne s'emparent de son âme à sa mort, bien qu'elle se confie « au grant deable d'enffer» (p. 49). Cette volonté d'effacer le surnaturel démoniaque laisse donc penser que le prosateur l'a jugé difficile à concilier avec la glorification du Chevalier 
au Cygne. C'est la seule modification d'importance qu'il apporte à la légende des origines de Godefroy de Bouillon, même s'il est ici encore moins innovant que l'auteur de la Chanson, puisque sa Matabrune conserve une fureur très impressionnante.

Avec l'autorité d'un moraliste, le prosateur du $\mathrm{XV}^{\mathrm{e}}$ siècle ne cesse ainsi de présenter l'histoire qu'il relate comme un récit exemplaire, un récit dont les leçons seraient intemporelles et donc toujours pertinentes pour les hommes et les femmes de son époque. Destinée non seulement à glorifier les origines du lignage de Clèves, mais aussi à transmettre des modèles de comportement, son œuvre est alourdie de gloses, de considérations psychologiques, éthiques ou politiques, au détriment d'une véritable recréation du texte-source. La Chanson en laisses épiques du XIV ${ }^{\mathrm{e}}$ siècle offre en revanche une réécriture inventive qui va bien au-delà d'une simple modernisation de la langue. Le souci d'une plus grande vraisemblance et la recherche de la transparence semblent toujours s'expliquer par un désir d'inventer une image plus prestigieuse des origines de la famille de Bouillon. Ainsi avons-nous analysé une transformation profonde du portrait des principaux personnages. Ce travail de recomposition permet de réhabiliter le père du Chevalier au Cygne, mis à mal dans Beatrix, d'effacer le surnaturel diabolique qui compromettait la grand-mère perverse Matabrune, toujours dans Beatrix, et aussi de rendre plus humains et plus pathétiques Elias et sa mère, tout en réintroduisant, sur le mode de la suggestion, les origines féeriques de cette dernière ${ }^{24}$. La célébration d'Elias comme chevalier de Dieu est bien entendu conservée, mais elle ne conduit pas l'auteur à lui attribuer des exploits contre les Sarrasins qui pourraient préfigurer ceux de Godefroy, comme si la croisade elle-même était réservée au petit-fils et à ses frères et que leurs exploits, inouïs, ne pouvaient supporter aucun précédent. L'explication de la réussite de la première Croisade que véhicule alors sur le mode de l'implicite la Chanson, c'est l'élection divine de l'ancêtre fondateur, un médiateur entre Dieu et le monde des hommes, qui, par sa nature même semble participer à la fois de l'humain et du surnaturel divin. D'après la Fin d'Elias, que réécrit aussi l'auteur du XIVe siècle, son départ signifie qu'il est « repris » par Dieu, puisqu'il se retire dans la solitude, après avoir réalisé la mission que Dieu lui a assignée dans le monde des hommes: non pas réussir la croisade - la mise en cycle ne serait plus possible avec les poèmes de la première Croisade -, mais fonder un lignage qui l'accomplira. Son destin s'inscrit alors en parallèle avec celui qui est fixé à sa mère dans Elioxe et la "concurrence » qui pouvait exister entre ces deux ancêtres fondateurs explique peut-être l'abrègement d'Elioxe et le rayonnement moindre accordé au personnage maternel. Pour l'auteur de la Chanson du XIV ${ }^{\mathrm{e}}$ siècle, la pratique de l'adaptation obéit donc à une véritable poétique de la métamorphose, alors que Berthault de Villebresme, malgré le choix d'une forme littéraire plus «moderne » et l'inscription de son œuvre dans le large mouvement des mises en prose du $\mathrm{XV}^{\mathrm{e}}$ siècle, ne procède pas à une relecture du texte-source qui lui permettrait de le refondre en profondeur, et que le "surplus » qu'il ajoute à Beatrix ne se caractérise pas par une grande originalité. 


\section{NOTES}

1. Voir The Old French Crusade Cycle, The University of Alabama Press, vol. I, La Naissance du Chevalier au Cygne, Elioxe, ed. E. J. Mickel, Beatrix, ed. J. A. Nelson, 1977 ; vol. II, Le Chevalier au Cygne and La Fin d'Elias, ed. J. A. Nelson, 1985 ; vol. III, Les Enfances de Godefroi and The Retour de Cornumarant, ed. E. J. Mickel, 1999. On ignore les dates précises et l'ordre exact de la composition des poèmes, qui émanent sans doute d'auteurs différents. La critique estime généralement que, pour la légende du Chevalier au Cygne, le plus ancien serait le Chevalier au Cygne et qu'il aurait été suivi par la Naissance du Chevalier au Cygne, puis par la Fin d'Elias. Voir S. Duparc-Quioc, Le Cycle de la croisade, Paris, Champion, 1955.

2. The Old French Crusade Cycle, vol. II, The Jerusalem Continuations, part 2, La Prise d'Acre, La Mort Godefroi and la Chanson des Rois Baudouin; vol. VIII, The Jerusalem Continuations, The London-Turin Version, éd. P. R. Grillo, The University of Alabama Press, 1987 et 1994.

3. Voir C. Gaullier-Bougassas, La Tentation de l'Orient dans le roman médiéval, Paris, Champion, 2003, et « Images littéraires de Chypre et évolution de l'esprit de croisade au XIV siècle », Progrès, réaction, décadence dans l'Occident médiéval, Genève, Droz, 2003, p. 123-135.

4. Sur cette légende, voir les études de C. Lecouteux, Mélusine ou le Chevalier au Cygne, Payot, 1982 et de L. Harf-Lancner, Les Fées au Moyen Âge, Morgane et Mélusine ou la naissance des fées, Paris, Champion, 1984.

5. Jean de Haute-Seille, Historia septem sapientum, Heidelberg, 1913; Herbert, le Roman de Dolopathos, éd. J.-L. Leclanche, Paris, Champion, 3 vol., 1997 (Cygni, v. 9203-10151).

6. G. Duby, Hommes et structures du Moyen Âge, Paris, 1973.

7. Voir les études déjà citées de L. Harf-Lancner et C. Lecouteux.

8. C. Gaullier-Bougassas, La Tentation de l'Orient dans le roman médiéval, op.cit.; "Images mythiques de l'Angleterre saxonne et création d'une mémoire collective : les romans de Waldef et de Gui de Warewic ", à paraître dans les actes du colloque de Versailles-Saint-Quentin, Le passé à l'épreuve du présent, 2005.

9. Godefroi de Buillon, The Old French Crusade Cycle, vol. X, ed. J. B. Roberts, The University of Alabama Press, 1996.

10. Éd. M. Golschmidt, Tübingen, 1899 ; C. Lachet, Sone de Nansay et le roman d'aventures en vers au XIII siècle, Paris, Champion, 1992.

11. C. Lecouteux, op. cit. ; Th. Cramer, Lohengrin, Munich, 1971 ; A. Kerdelhé, Lohengrin, Kümmerle Verlag, Göppingen, 1986.

12. Le Mythe de croisade, Paris, Gallimard, 1997, 4 vol.

13. La Chanson du Chevalier au Cygne et de Godefroy de Bouillon, éd. F. de Reiffenberg, Bruxelles, $3 \mathrm{t}$., 1846-1859. Sur le deuxième cycle de la croisade, voir S. Duparc-Quioc, Le Cycle de la croisade, op. cit. et "Les poèmes du $2^{\mathrm{e}}$ cycle de la croisade : problèmes de composition et de chronologie ", Revue d'histoire des textes, 9, 1979, p. 141-181; R. Cook et L. S. Crist, Le deuxième cycle de la croisade, Genève, Droz, 1972. La mise en prose du XV siècle a été éditée par E. A. Emplaincourt, La Geste du Chevalier au Cygne, The Old French Crusade Cycle, vol. IX, The University of Alabama Press, 1989. Voir aussi E. A. Emplaincourt et J. A. Nelson, « La Geste du Chevalier au Cygne : la version en prose de Copenhague et la tradition du premier cycle de la croisade », Romania, 105, 1983, p. 351-370.

14. Voir F. de Reiffenberg, éd. cit., t. I, p. xxiii-xxxiv et H. Pigeonneau, Le Cycle de la croisade et la famille de Bouillon, Saint-Cloud, 1877.

15. Mémoires d'olivier de la Marche, Paris, éd. H. Beaune et J. d'Arbaumont, S. H. F., 1873-1878, t. II, p. 342.

16. Op. cit., p. 345-346.

17. Ibidem, p. 351 . 
18. C. Gaullier-Bougassas, La Tentation de l'Orient..., op. cit., p. 289-354.

19. F. Suard, «Pierre Desrey et la Genealogie de Godefroy de Bouillon », Chanson de geste et tradition épique en France au Moyen Âge,Orléans, Paradigme, 1994, p. 387-398. Voir aussi L. S. Crist, « Pierre Desrey et son histoire de la croisade », Mélanges J. Lods, Paris, 1978, p. 153-172, et G. Doutrepont, «La légende du Chevalier au Cygne pendant le XVI siècle », Mélanges A. Franc, Paris, Droz, 1936, p. 26-36.

20. Sur les réécritures du conte des enfants-cygnes au XIII ${ }^{\mathrm{e}}$ siècle, voir G. Paris, «La Naissance du Chevalier au Cygne", Romania, 19, 1890, p. 314-340 ; F. Lot, "Le mythe des enfants-cygnes", Romania, 21, 1892, p. 52-67 ; J. Lods, "L'utilisation des thèmes mythiques dans trois versions écrites de la légende des enfants-cygnes", Mélanges R. Crozet, Poitiers, 1966, t. 2, p. 809-820, «Encore la légende des enfants-cygnes: version courtoise et version pseudo-épique, étude de style », Mélanges R. Lejeune, Gembloux, Duculot, 1969, t. 2, p. 1227-1244, «L'art de la composition dans les différentes versions du Chevalier au Cygne ", Studia Romanica, 14, 1969, p. 245-263 ; W. R. J. Barron, "Versions and texts of the Naissance du Chevalier au Cygne", Romania, 89, 1968, p. 481-538; C. Roussel, «Le conte et le mythe, histoire des enfants-cygnes ", Frontières du conte, Paris, CNRS, 1982, p. 15-24 ; C. Lecouteux, Mélusine et le Chevalier au Cygne, op. cit.; A. Kerdelhué, "Les métamorphoses de l'eau dans trois versions de la Naissance du Chevalier au Cygne ", Les quatre éléments de la culture médiévale, Kümmerle Verlag, 1983, p. 127-140 ; L. Harf-Lancner, Les Fées..., op. cit., p. 179-198 ; D. A. Trotter, «L'ascendance mythique de Godefroy de Bouillon et le cycle de la croisade », Métamorphose et bestiaire fantastique au Moyen Âge, Paris, 1985, p. 107-135 ; J. FoehrJanssens, Le Temps des fables. Le Roman des Sept Sages, ou l'autre voie du roman, Paris, Champion, 1994, notamment p. 255-266.

21. Voir L. Harf-Lancner, «La métamorphose illusoire: des théories chrétiennes de la métamorphoses aux images médiévales du loup-garou ", Annales, 1985, 1, p. 208-226.

22. Elioxe apportait quant à elle des richesses extraordinaires - pierres du Paradis, onguents magiques -, même si ces trésors ne jouaient aucun rôle dans la suite du récit (v. 284-323).

23. Dans Beatrix, Matabrune exige que le serviteur rapporte les chaînes et elle n'envisage la possibilité de l'assassinat des enfants que s'il ne parvient pas à les dérober (v. 439-443).

24. L'auteur modifie bien davantage Beatrix qu'Elioxe. Il ne s'inspire certes que du début d'Elioxe, mais ce poème offrait déjà des portraits du père, de la mère et de la grand-mère d'Elias en meilleure adéquation avec le projet de glorification du lignage, bien qu'Elias n'en fût pas le héros principal.

\section{AUTEUR}

\section{CATHERINE GAULLIER-BOUGASSAS}

Université Lille-III 